\title{
PROCESOS DE PIPING EN LA REGIÓN DE MURCIA (SURESTE DE ESPAÑA)
}

\author{
A. ROMERO DÍAZ ${ }^{(1)}$, P. MARÍN SANLEANDRO ${ }^{(2)}$ y A. SÁNCHEZ SORIANO ${ }^{(1)}$
}

(1) Departamento de Geografía. Universidad de Murcia. Campus de La Merced, 30001-Murcia

(2) Departamento de Química Agrícola, Geología y Edafología. Universidad de Murcia. Campus de Espinardo, 30100 Espinardo-Murcia correo electrónico de contacto: arodi@um.es

RESUMEN. Los procesos de erosión subsuperficial o piping tienen un amplio desarrollo en medios semiáridos cuando se dan las características idóneas para ello. En la Región de Murcia se han localizado 67 áreas donde la presencia de piping afecta a una superficie bastante notable y cuyo grado de evolución está muy avanzado. Estas áreas suelen coincidir con cuencas neógenas-cuaternarias, de litología margosa, y cuyo uso del suelo predominante son antiguos campos de cultivos de secano, aterrazados y hoy en abandono.

En todas las áreas se han realizado detallados estudios morfológicos y en 15 de ellas se tomaron muestras de suelo a dos profundidades (superficie y $100 \mathrm{~cm}$ de profundidad). Se han realizado análisis físico-químicos con el objetivo de estudiar sus características y establecer posibles causas de formación.

Desde el punto de vista morfológico destaca la importancia del aterrazamiento al generar un gradiente hidráulico y como la profundidad de los pipes suele coincidir con la altura de la terraza inferior. Los resultados de los análisis realizados muestran como la presencia de piping, en la mayoría de las áreas, se encuentra ligada a cambios en la estructura y textura de los suelos a distintas profundidades (texturas mas finas en profundidad y suelos carentes de estructura), y elevados porcentajes de sodio de cambio (superiores a niveles más profundos), lo que favorece la dispersión del suelo y la aparición de estos procesos. Como prácticas de conservación del suelo, en áreas con estas características, sería totalmente desaconsejable el aterrazado de los campos, en particular con terrazas altas, y su abandono posterior.

ABSTRACT. The processes of sub superficial erosion or piping have a wide development in semiarid environment when the suitable characteristics for it happen. In the Region of Murcia 67 areas have been located where the presence of piping concerns a notable surface and whose evolution degree is very 
advanced. These areas often coincide with neogene-quaternary basins, of marly lithologie, and whose predominant soil use are old dryness culture fields, terraced and abandon today.

In all the areas detailed morphologic studies have been realized and in 15 of them, soil samples at two depths (surface and $100 \mathrm{~cm}$ depth) were taken. Physic - chemist analysis have been carried out with the aim to study their characteristics and to establish possible formation reasons.

From the morphologic point of view, the terraced importance stands out on having generated a hydraulic gradient and as the pipes depth usually coincides with the low terrace height. The results of the realized analyses show as the piping presence, in the majority of the areas, is linked to changes in the structure and soils texture at different depths (thinner textures in depth and lacking structure soils), and high exchangeable sodium percentage (superior at deeper levels) what favours the soil dispersion and the appearance of these processes.

As practices of soil conservation, in areas with these characteristics, the terraced fields would be totally inadvisable, especially with high terraces, and its later abandon.

Palabras clave: piping, margas, cárcavas, erosión, abandono de tierras, región de Murcia, medios semiáridos.

Key words: piping, marls, gullies, erosion, abandonment, region of Murcia, semiarid environment.

\section{Introducción y antecedentes}

El término piping (erosión en tunel), definido por numerosos autores (Jones, 1981), se usa para describir los procesos de erosión subsuperficiales. El proceso se origina por flujos subsuperficiales concentrados que comienzan a partir de grietas de desecación o pequeñas diaclasas y provocan la remoción y disolución del material, creando conductos tubulares subterráneos (pipes), que evolucionan, en ocasiones, hacia cárcavas profundas de paredes verticales.

Las causas que originan el piping, descritas ampliamente, pueden ser de diverso origen: mecánicas, químicas, o bióticas. De entre las causas esgrimidas con mayor frecuencia están: la existencia de un gradiente hidráulico, diferencias de porosidad-permeabilidad en los distintos horizontes del suelo, presencia abundante de sodio y el tipo de uso del suelo (Parker, 1964).

El piping se ha observado tanto en paisajes naturales como antrópicos, en diferentes climas, litologías y depósitos, así como bajo diferentes usos del suelo y cubierta vegetal (Parker, 1964; Heede, 1971; Jones, 1981; Bryan y Jones, 1997; Faulkner, 2006). No obs- 
tante, en medios semiáridos alcanza un gran desarrollo y es donde se encuentran las formas mayores (Bryan y Jones, 1997). En España, los estudios realizados sobre piping se centran en la Depresión del Ebro (Mensua e Ibáñez, 1975; Gallart, 1979; Rodríguez Vidal, 1982; Gracia Prieto, 1986; Gutierrez y Rodríguez, 1984; Gutierrez et al., 1988, 1994, 1997; Del Valle y Del Val, 1990; Barrón et al., 1994; García Ruiz et al., 1986, 1994, 1997), el Centro Peninsular (Ternan et al., 1998), y el Sureste de España, en las provincias de Alicante, Almería y Murcia (Harvey, 1982; Calvo et al., 1991; Calvo y Harvey, 1996; López Bermúdez y Torcal Sainz, 1986; López Bermúdez y Romero Díaz, 1989; Martín Penela, 1990, 1994, Ferre Bueno et al., 1994; Watts, 1991; Vandekerckhove et al., 2000; Poesen et al., 2002; Faulkner et al., 2000, 2003a, 2003b, 2006; Romero Díaz et al., 2007).

En la Región de Murcia, los procesos de erosión por piping tienen una amplia representación. Trabajos anteriores ya pusieron de manifiesto la existencia de áreas con piping en la región. Harvey (1982) los identificaba en Sucina (Murcia), aunque en la actualidad transformaciones humanas del terreno los han hecho desaparecer; Romero Díaz y López Bermúdez (1985) hablaban de ellos en la Cuenca de Mula, en los alrededores de Campos del Río; López Bermúdez y Torcal Sainz (1986) localizan otra área en las cercanías de Mazarrón; López Bermúdez y Romero Díaz (1989) representaban en un mapa del Sureste peninsular, algunas áreas con procesos de piping en la Región; Arana et al. (1992) citan un sector próximo a Fuente Librilla con existencia de procesos de piping y, más recientemente, Vandekerckhove et al., (2000) y Poesen et al., (2002) estudian en la cabecera del río Guadalentín un área con presencia importante de estos procesos. En la cuenca de Mula, en los últimos años, se ha realizado un estudio exhaustivo de este proceso (Marín Sanleandro el al., 2004; Sánchez Soriano et al., 2003, 2004; Romero Díaz et al., 2007).

Aquí se analizan distintas áreas de la Región de Murcia, en las cuales los procesos de piping están muy extendidos. En ellas se han realizado análisis físico-químicos de los suelos para poder establecer algunas de sus causas de formación.

\section{2. Áreas de estudio}

En el estudio que, en la actualidad, se está llevando a cabo, se han localizado 67 áreas (tabla 1), en las cuales el desarrollo de los procesos de piping es bastante importante. Las principales áreas se encuentran distribuidas por toda la región (Fig. 1) y suelen coincidir con áreas de margas donde las cárcavas, en mayor o menor medida, suelen estar presentes.

Las áreas con más densidad de pipes se localizan en las cuencas neógenas-cuaternarias de Mula y Fortuna-Abanilla. No obstante, numerosos ejemplos de ellos se han localizado en los términos municipales de Cieza, Molina de Segura, Murcia, Librilla, Alhama, Lorca y Mazarrón. Es de mencionar como los procesos de piping incipientes o con fenómenos aislados de pipes son muy abundantes en numerosos puntos de la región, y que evidentemente, no todas estas zonas han sido contabilizadas en este inventario; si hubiera sido así, su número sería infinitamente mayor. 
Tabla 1. Localización de las principales áreas de piping en los MTN escala 1:50.000

\begin{tabular}{|l|c|}
\hline MTN 1:50.000 & Áreas con piping \\
\hline Calasparra 890 & 4 \\
Cieza 891 & 1 \\
Fortuna 892 & 13 \\
Mula 912 & 17 \\
Orihuela 913 & 7 \\
Alhama 933 & 4 \\
Murcia 934 & 6 \\
Velez Blanco 952 & 6 \\
Lorca 953 & 6 \\
Mazarrón 976 & 3 \\
\hline Total & $\mathbf{6 7}$ \\
\hline
\end{tabular}

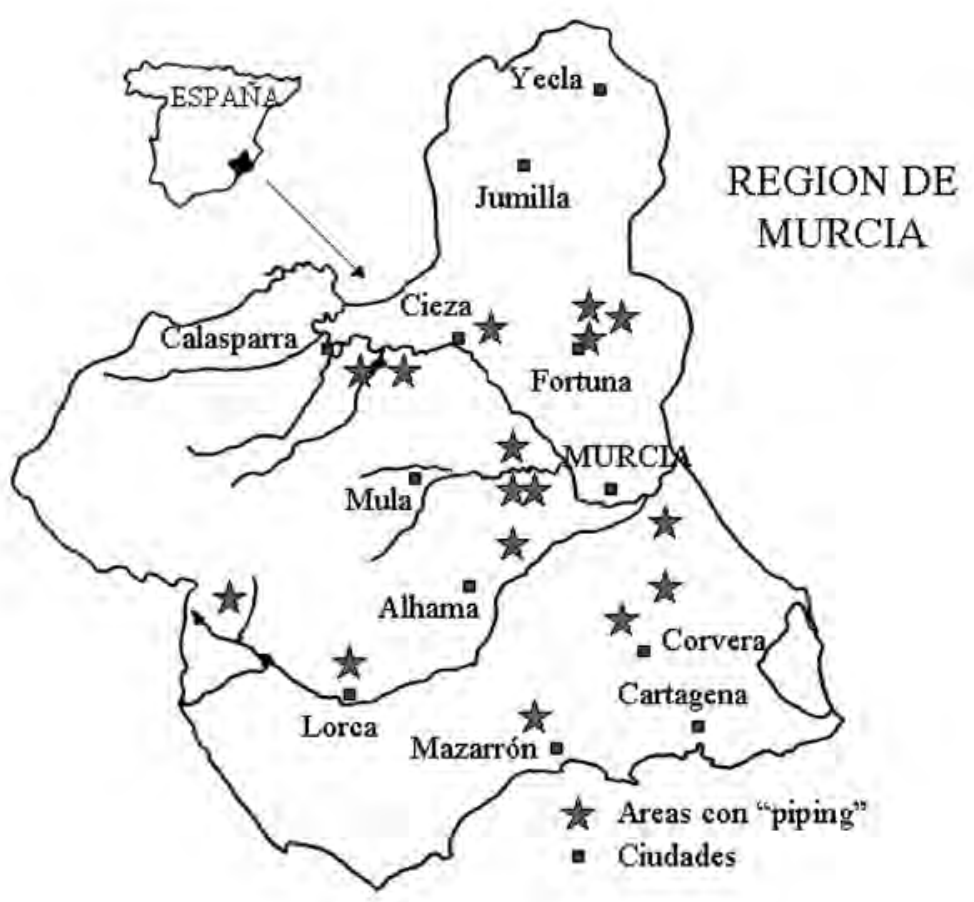

Figura 1. Localización de las principales áreas con "piping” en la Región de Murcia 
Las características climáticas de la mayor parte de la Región de Murcia son mediterráneas semiáridas, con una temperatura media de $17^{\circ} \mathrm{C}$ y unas precipitaciones medias anuales en torno a los $300 \mathrm{~mm}$, pero con una elevada irregularidad y con frecuentes episodios de alta intensidad.

Los suelos mayoritarios en las áreas de piping son Regosoles calcáricos (FAO, 1999), desarrollados sobre margas y afectados por salinidad. La escasa vegetación actual existente (en el mejor de los casos) se corresponde con un matorral muy degradado, de escaso porte e insuficiente carácter protector de los suelos; de entre las especies más frecuentes destacan: Salsola genistoides, Lygeum spartum, Asparagus, Moricandia arvensis, Thimelaea hirsuta y Artemisia herbaalba.

\section{Métodos}

La identificación de las áreas de piping en la Región de Murcia se ha realizado mediante exhaustivos reconocimientos de campo. Previo a ellos se hizo un estudio de fotointerpretación de toda la Región, usando las fotografías aéreas en formato digital del Instituto Geográfico Nacional del vuelo de 1999. La posible identificación de áreas con piping se localizaron en los mapas del mismo organismo a escala 1:25.000, que han servido de guía para los trabajos de campo. En ocasiones, las transformaciones recientes efectuadas por el hombre, con grandes movimientos de tierras, han hecho desaparecer áreas con piping, que en la fotografía aérea de 1999 aparecían con bastante claridad. Por el contrario, en áreas abandonadas recientemente aparecen en el campo procesos de piping que en la fotografía aérea, por su carácter incipiente o inexistencia en este año, son imposibles de observar.

En el campo, en las principales áreas estudiadas, se han realizado diversas observaciones y mediciones de sus características morfológicas. En 15 áreas representativas se tomaron muestras de suelo a dos profundidades: de 0 a $30 \mathrm{~cm}$ (identificadas como A) y a $100 \mathrm{~cm} \mathrm{de}$ profundidad (identificadas como B) (tabla 2). Con posterioridad, en el laboratorio se analizaron sus características, tanto físicas como químicas, para poder establecer diferencias y similitudes entre las distintas áreas, al mismo tiempo que investigar las causas de su formación.

En laboratorio se ha realizado el análisis granulométrico (siguiendo el método de Robinson); consistencias (en seco, húmedo y mojado) (FAO, 1977), estabilidad de agregados (Lax et al., 1994); y se han determinado distintos parámetros como: conductividad eléctrica (Bower y Wilcox, 1965), iones en el extracto de saturación por cromatografía iónica, pH (Peech, 1965), cantidad de carbonato cálcico total (calcímetro de Bernard), materia orgánica (Anne, 1945; Duchaufour, 1970), capacidad de cambio catiónico (Chapman, 1965), etc.

Para el estudio de la mineralogía de la fracción arcilla, se ha utilizado la difracción de rayos $\mathrm{X}$, habiéndose obtenido los diagramas correspondientes a las muestras en agregado orientado, tras someterlos a diversos tratamientos después de la saturación con magnesio, solvatación con etilénglicol y tratamiento térmico. También se ha realizado la saturación en potasio. La estimación semicuantitativa relativa se ha efectuado teniendo en cuenta los poderes reflectantes dados por Martín Pozas et al. (1969). 


\begin{tabular}{|c|c|c|c|c|c|c|c|c|c|c|c|}
\hline हैं & is & is & is & is & is & is & $\stackrel{\circ}{z}$ & is & $\stackrel{0}{z}$ & is & is \\
\hline $\begin{array}{l}\frac{\pi}{00} \\
\frac{0}{9} \\
\frac{0}{3}\end{array}$ & 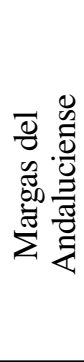 & 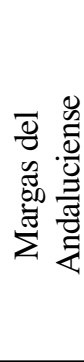 & 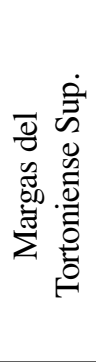 & 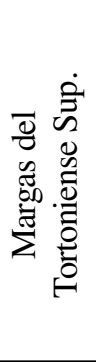 & 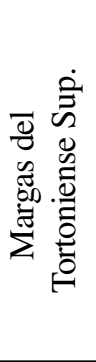 & 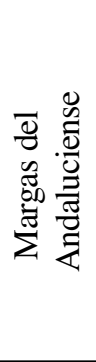 & 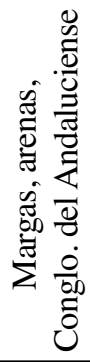 & 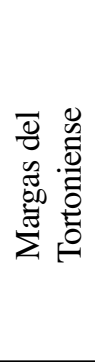 & 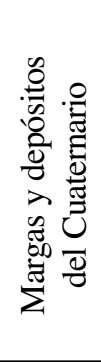 & 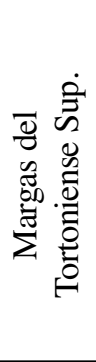 & 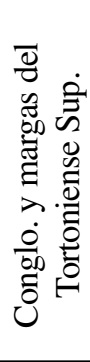 \\
\hline $\begin{array}{l}\frac{0}{0} \\
\frac{0}{0} \\
\frac{\partial}{0} \\
0 \\
0 \\
0 \\
0\end{array}$ & 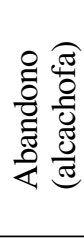 & 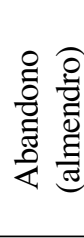 & 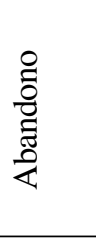 & 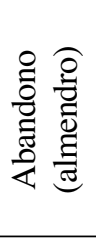 & 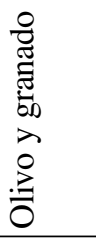 & 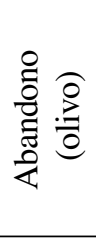 & U் & 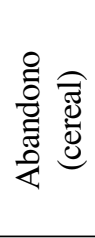 & 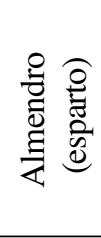 & 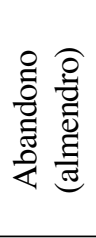 & 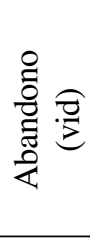 \\
\hline 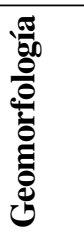 & 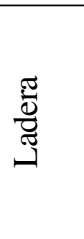 & $\frac{\pi}{0}$ & 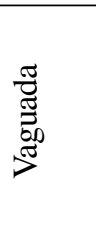 & $\begin{array}{l}\frac{\pi}{\tilde{\Xi}} \\
\stackrel{\Xi}{\tilde{0}} \\
\frac{0}{<}\end{array}$ & 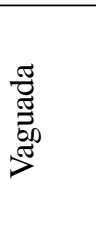 & 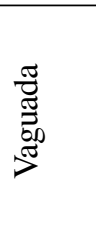 & $\frac{\frac{n}{\pi}}{\sqrt[\pi]{0}}$ & 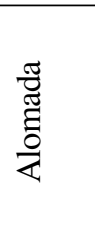 & $\frac{\pi}{0}$ & 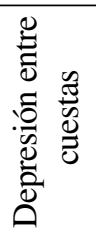 & $\frac{\frac{n}{0}}{\frac{\pi}{0}}$ \\
\hline 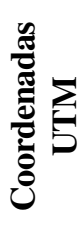 & 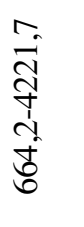 & $\begin{array}{l}\infty \\
\frac{\infty}{2} \\
\frac{1}{1} \\
\stackrel{8}{8}\end{array}$ & 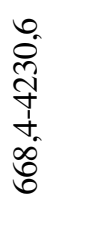 & 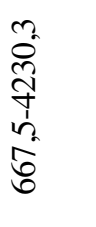 & 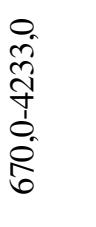 & 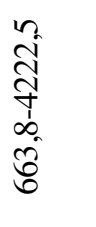 & 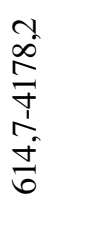 & 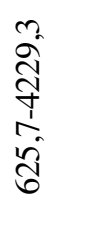 & 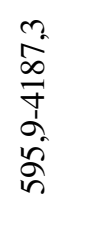 & 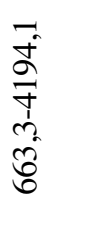 & $\begin{array}{l}n \\
\infty \\
0 \\
+ \\
\vdots \\
0 \\
0\end{array}$ \\
\hline 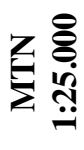 & ๗̀ & $\stackrel{\vec{n}}{\sigma}$ & $\begin{array}{l}\text { 䘚 } \\
\text { న̆ }\end{array}$ & $\begin{array}{l}\text { 䘚 } \\
\text { న⿱ }\end{array}$ & 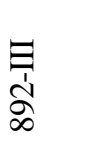 & $\stackrel{\vec{n}}{\sigma}$ & 貝 & $\frac{7}{8}$ & 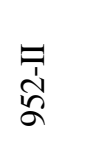 & $\frac{\Xi}{\not}$ & $\frac{\sqrt{1}}{\frac{1}{a}}$ \\
\hline 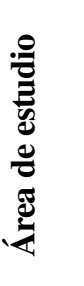 & 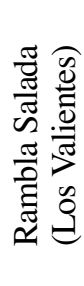 & 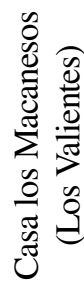 & 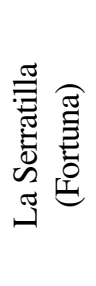 & 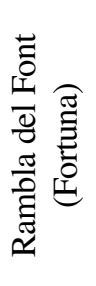 & 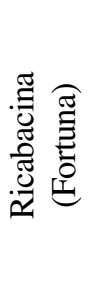 & 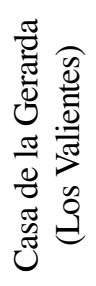 & 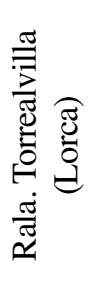 & 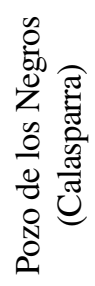 & 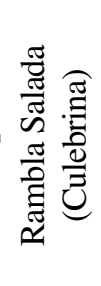 & 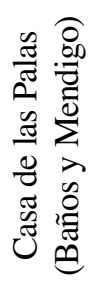 & 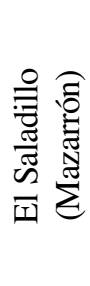 \\
\hline 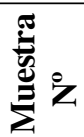 & - & $N$ & $m$ & $\nabla$ & $n$ & 0 & $r$ & $\infty$ & $a$ & $\stackrel{ }{\circ}$ & $=$ \\
\hline
\end{tabular}




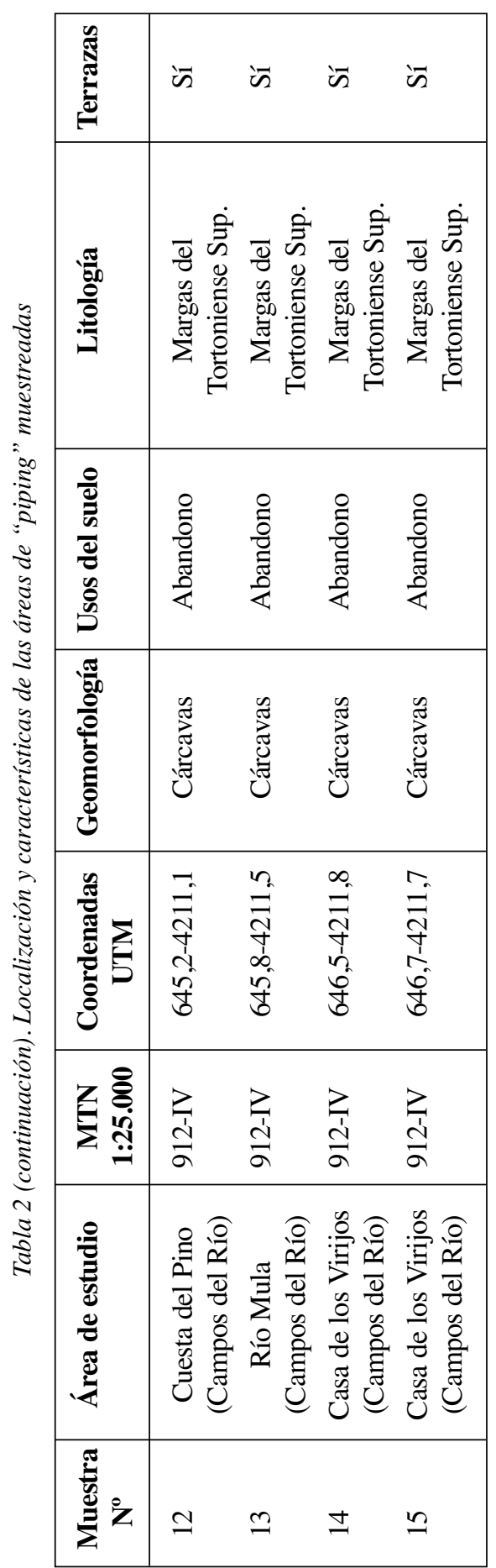




\section{Resultados y discusión}

\subsection{Características generales de las áreas afectadas por piping}

La mayoría de las zonas con piping se han desarrollado sobre margas del Tortoniense y del Andaluciense y en algunos casos, junto con las margas, aparecen arenas y conglomerados. En el 70\% de los casos el piping aparece ligado a campos de cultivo aterrazados (en su mayoría abandonados) y el resto a áreas de badland y cabeceras de ramblas. En el caso de los procesos de piping en campos de cultivo abandonados, su desarrollo y evolución está relacionado con los años de abandono.

Las profundidades más elevadas se encuentran en áreas con cárcavas y cabeceras de ramblas, 15 metros en el Paraje de La Murta (Calasparra) y 12 metros en la Rambla de Periago (Cabecera del Guadalentín). En campos aterrazados la profundidad está relacionada con la altura de las terrazas de cultivo (en torno a 2-3 metros), aunque se han encontrado pipes de 7-8 metros, cuando dos terrazas se han puesto en comunicación. La diferencia de altura entre parcelas constituye un parámetro muy importante pues determina, en la mayoría de los casos, la profundidad de los pipes, siendo la parcela inferior el nivel de base de la superior (Fig. 2). Se constata aquí, como ya ha sido hecho por otros muchos autores, la importancia del gradiente hidráulico como una de las causas generadoras de los pipes (Barrón et al., 1994). En la mayoría de los casos, los pipes se presentan alineados e interconectados, tanto entre los existentes en una misma parcela como entre parcelas, habiéndose generado una nueva red de drenaje, como la que debió existir antes del aterrazado y puesta en cultivo de las vaguadas.

La escasa productividad de la mayoría de las tierras de secano de la Región de Murcia y los procesos de erosión hídrica que afectan a estos suelos, lo que supone un eleva-

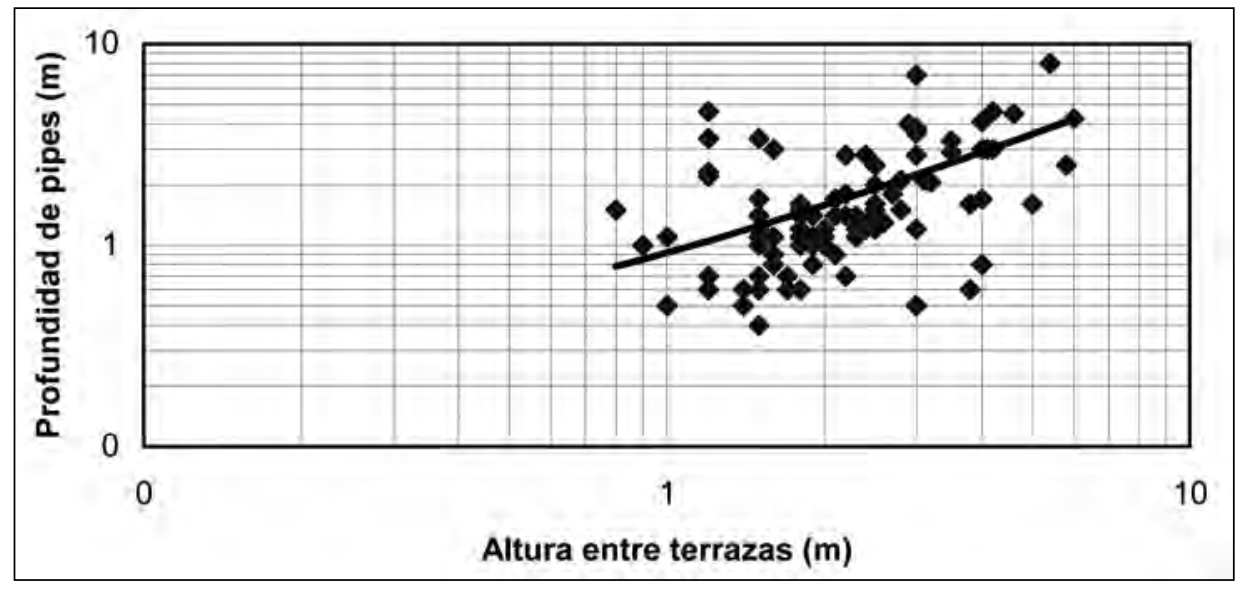

Figura 2. Relación entre la altura de las terrazas y profundidad de pipes en 142 parcelas medidas en el área de Campos del Río 
do coste de mantenimiento de las parcelas de cultivo, son las razones fundamentales de su abandono (Romero Díaz, 2002). Tras el cual, la aparición y/o evolución de los procesos de piping, en las tierras susceptibles para ello, se produce de forma inmediata.

La variedad de formas y grados de evolución de los pipes es grande. A grandes rasgos en la región de Murcia se pueden distinguir dos tipos: (1) los pipes asociados a badland y (2) los pipes desarrollados en campos de cultivo abandonados.

Los primeros se localizan principalmente en las cabeceras de los barrancos, contribuyendo de una manera eficaz a la erosión remontante del mismo. Las profundidades suelen ser elevadas, en especial en aquellos lugares donde el desnivel topográfico es grande. Buenos ejemplos de este tipo de pipes los encontramos en las ramblas de cabecera de la cuenca del Guadalentín (Fig. 3) y otras ramblas que vierten al río Segura (Fig. 4).

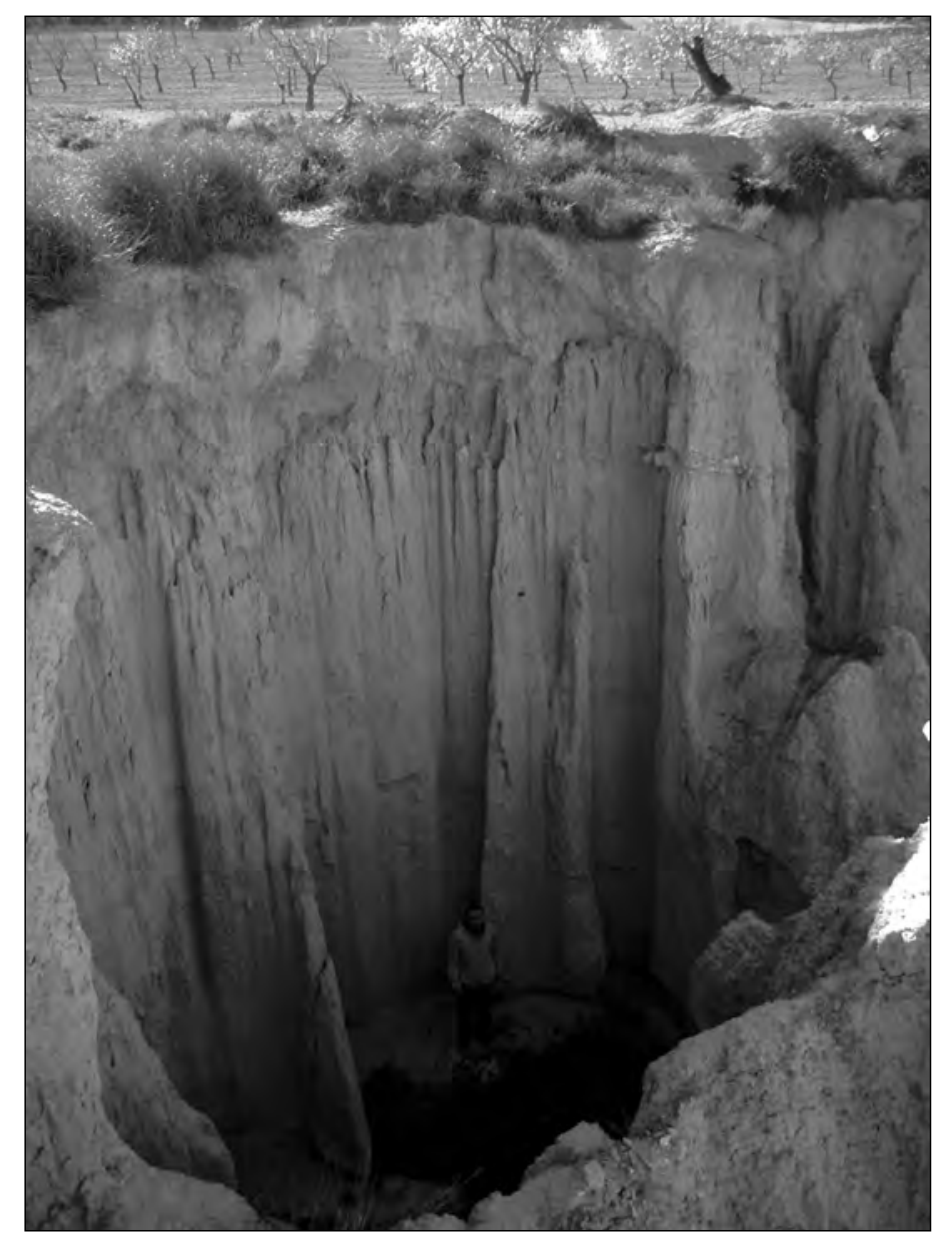

Figura 3. Rambla de Torrealvilla (Lorca) 


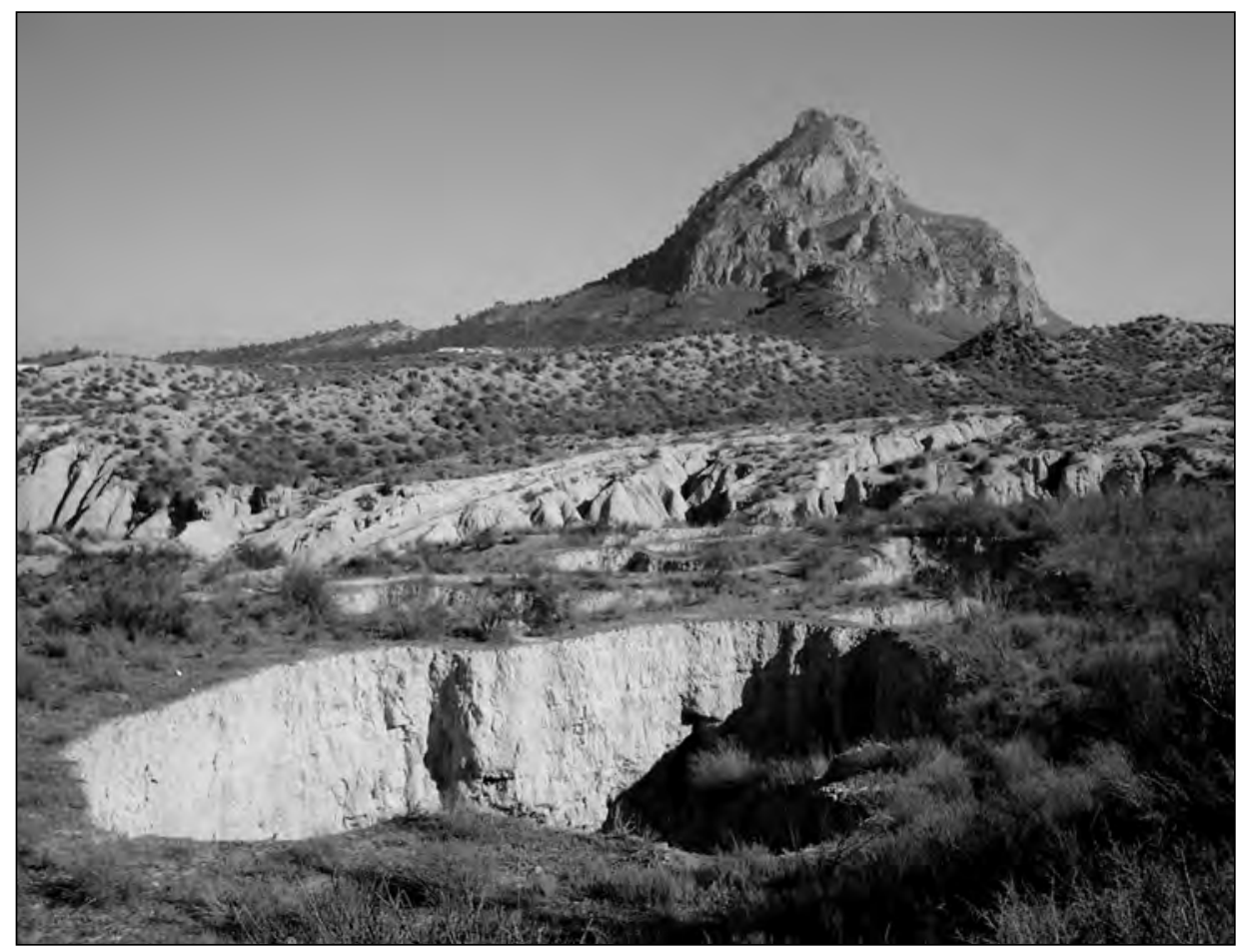

Figura 4. Paraje de La Murta (Calasparra)

Los segundos ocupan amplias superficies en campos aterrazados para el cultivo (de cereal, almendro y olivar) y con posterioridad abandonados. El grado de evolución y desarrollo de las áreas de piping dependen del tiempo de abandono de los campos. Las áreas con mayor desarrollo de este tipo de pipes se han localizado en las vaguadas de las ramblas que vierten al río Mula (términos municipales de Albudeite y Campos del Río), diversos sectores en la cuenca de Fortuna.Abanilla y en un área localizada entre El puerto de La Cadena y la localidad de Corvera (Fig. 5).

En campos abandonados hace unos cuantos años, el proceso es incipiente. Por el contrario, en campos abandonados de más de 40-50 años, se ha instalado una nueva red de drenaje, conectando todas las antiguas terrazas en una red jerarquizada y ofreciendo un aspecto de paisaje en badland, pero en esta ocasión originado por causas antrópicas.

\subsection{Propiedades físicas de los suelos}

Las propiedades físicas de los suelos pueden explicar algunas de las causas que contribuyen o favorecen la formación de los procesos de piping, En particular, se ha analizado su consistencia, estructura, estabilidad de agregados, textura y mineralogía de arcillas. 


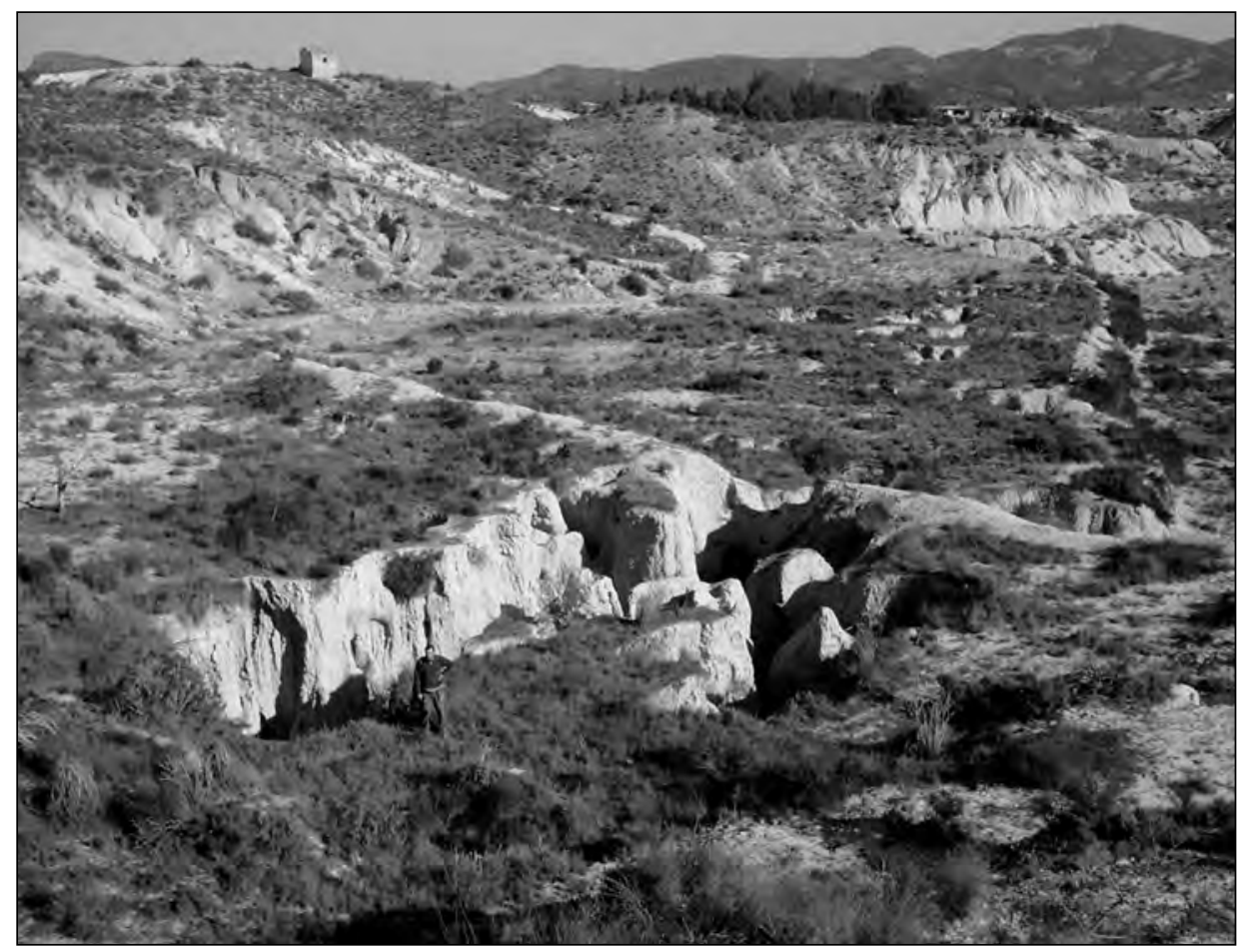

Figura 5. Los Jurados (Corvera)

Desde el punto de vista de la consistencia, los suelos estudiados en seco suelen ser blandos o ligeramente duros y en mojado muy adherentes y plásticos (tabla 3).

Una de las propiedades edáficas que permiten diferenciar un suelo del material geológico es la estructura. Esta propiedad física expresa la distribución espacial y la organización de las partículas del suelo (Hillel, 1980) y condiciona parámetros edáficos tan importantes como la circulación del aire y el agua, determinante en el proceso de piping. Por lo general, los horizontes A de estos suelos tienen una estructura poliédrica subangular mediana y gruesa débil (FAO, 1977) mientras que en profundidad se presentan masivos y compactados, prácticamente sin estructura. Esta desestructuración es precisamente la responsable de que el proceso de piping comience en estos niveles más profundos. La falta de estructura se explica por el complejo arcillo-húmico poco estable, debido a la falta de humus, a sus pHs altos y elevados porcentajes de sodio de cambio, que provocan la dispersión de la fracción arcilla, muy abundante en la marga. Mientras que varios autores argumentan que el sodio sobre el complejo de la arcilla dispersa la fracción arcilla formando un suelo desprovisto de complejo arcillo-húmico muy erosivo, Robinson y Phillips (2001), así como Mualem y Assouline (1992), opinan que el hinchamiento de las arcillas es la causa de la dispersión, de modo que con un alto porcentaje el efecto de hinchamiento restringe la infiltración y el desarrollo de pipes. Pero, cuando los porcentajes de arcilla son inferiores al 70\%, la dispersión asociada con la acción del sodio desagrega la estruc- 
tura y entonces no disminuye la infiltración. Con la presencia de un gradiente hidráulico estas condiciones son óptimas para el desarrollo de pipes (Romero Díaz et al., 2007).

La estabilidad estructural es una cualidad edáfica derivada de la estructura del suelo. Se define como la fuerza de unión entre las partículas elementales de los agregados del suelo, siendo de gran importancia en la protección contra la pérdida de suelo por erosión (Cerdá, 1998). Las muestras en las que se ha analizado este parámetro (Lax et al., 1994) presentan valores inferiores al 20\%, pudiéndose considerar pobremente estructurados, lo que contribuye a favorecer el proceso de formación de pipes; además la estabilidad de agregados suele disminuir con la profundidad. En muy pocos casos se ha alcanzado un $70 \%$ de agregados estables, teniendo una estructura que se puede considerar casi buena (Sánchez Soriano et al., 2004). De esta baja estabilidad de agregados puede responsabilizarse la escasez de materia orgánica (Gerzabek et al., 1995) y la dispersión del sodio sobre la fracción arcilla, como se ha comentado anteriormente.

Otro factor importante, junto a la textura arcillo-limosa, que contribuye a la formación de agregados en nuestros suelos son los carbonatos. El elevado contenido en carbonato cálcico refuerza el grado de compactación de los materiales finos, al mismo tiempo que ralentiza los procesos de meteorización química y favorece la mineralización de la escasa materia orgánica fresca que reciben. La mayoría de los suelos con desarrollo de piping son suelos muy calizos, lo que está en concordancia con la naturaleza del material original, margas constituidas por carbonato cálcico y arcilla. La disminución de los carbonatos en profundidad justifica también la menor estructuración del suelo a estos niveles y su contribución a la formación de pipes.

En cuanto a la textura del suelo, ésta tiene una gran importancia en relación con la impermeabilidad, la aireación y otras propiedades que favorecen la aparición de pipes. Algunas de la muestras, debido a la gran cantidad de sales, no dispersaron adecuadamente para poder realizar las extracciones de las fracciones limo y arcilla. Sí que se pudo aislar por tamización su fracción arena y conocer la suma de las fracciones limo y arcilla, pero no concretar el porcentaje de cada una de ellas. Todos los suelos analizados son suelos de textura muy fina con un carácter limo arcilloso o arcillo limoso, según predominen limo o arcilla respectivamente (tabla 3). En todos los pipes muestreados la textura es más fina en profundidad que en superficie (aspecto ya detectado por López Bermúdez y Romero Díaz en 1989 en un área de Campos del Río y por Faulkner et al. en el 2000 en Almería). Se presentan porcentajes más elevados de arcilla y limo fino a 100 $\mathrm{cm}$ (figura 6) lo que contribuye, en buena parte, a empeorar sus propiedades físicas y a facilitar la aparición de los túneles.

Con el objeto de estimar la influencia que tiene la mineralogía de arcillas de los suelos en el proceso de formación de pipes, en un área seleccionada (Campos del Río) se muestrearon pipes, a dos profundidades y se estudió la mineralogía de su arcilla.

La composición mineralógica de la fracción arcilla resultó ser bastante homogénea en las muestras tomadas, siendo illita y esmectitas los minerales más abundantes, a los que acompañan clorita, caolinita y muy pequeñas cantidades de cuarzo y feldespatos. La 


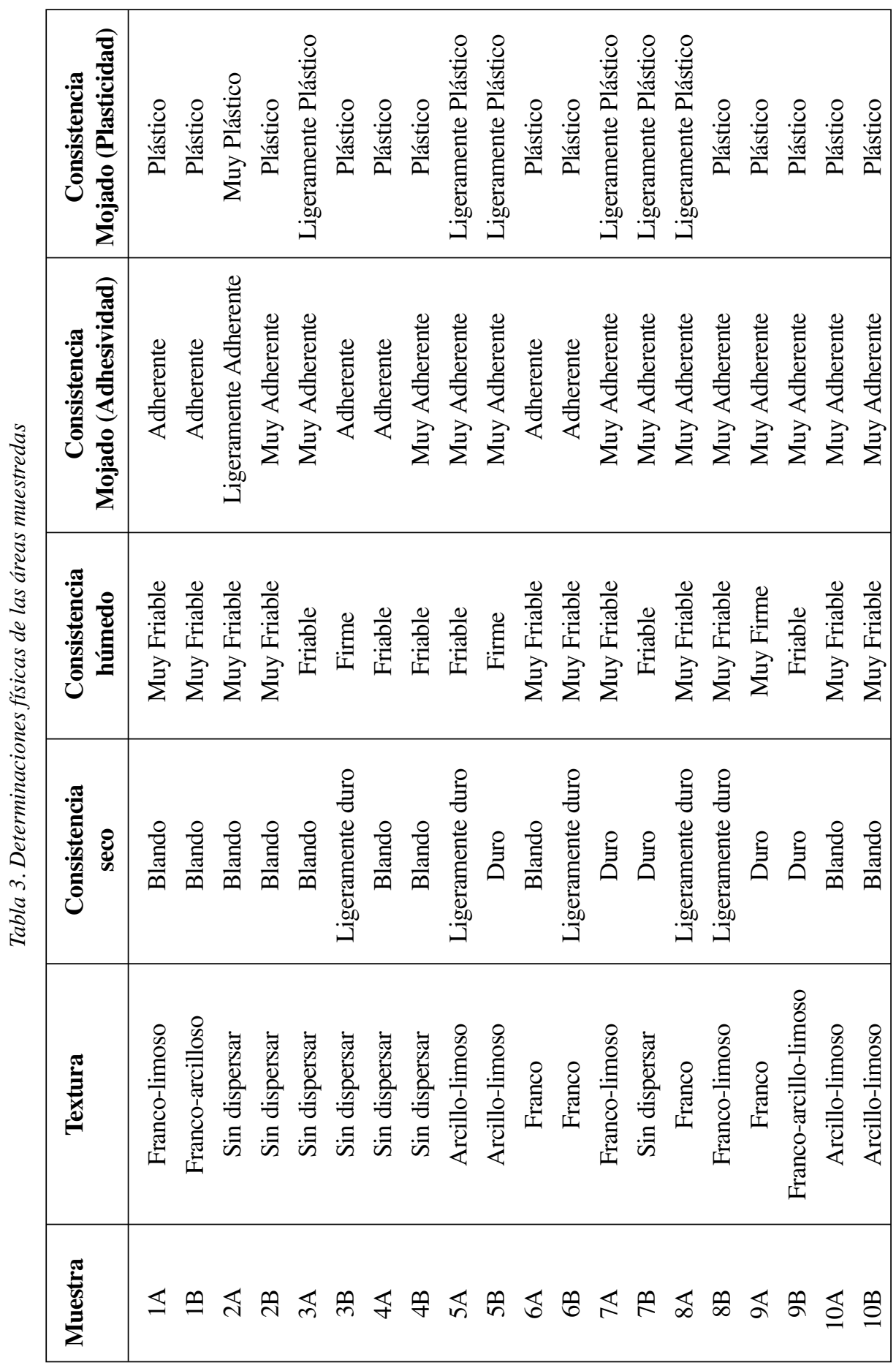




\begin{tabular}{|c|c|}
\hline 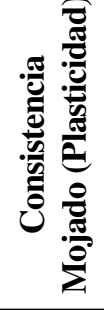 & 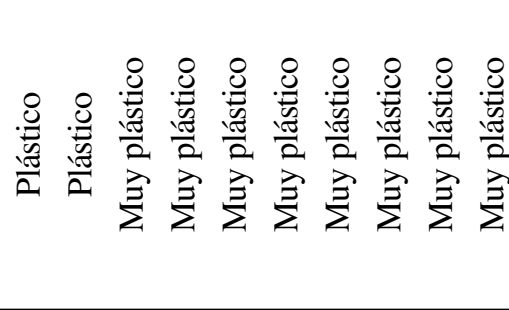 \\
\hline 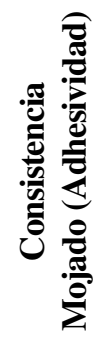 & 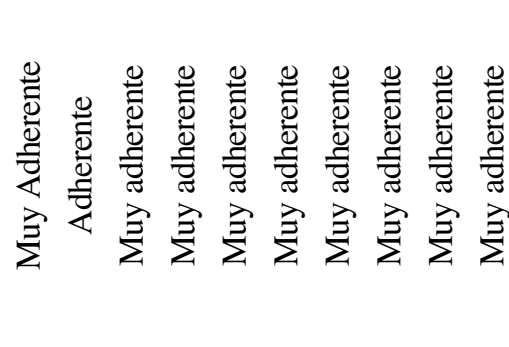 \\
\hline 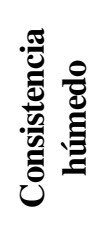 & 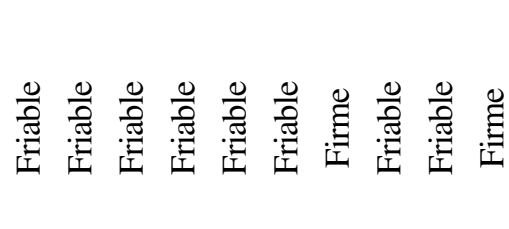 \\
\hline 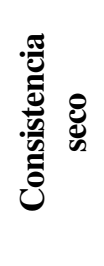 & 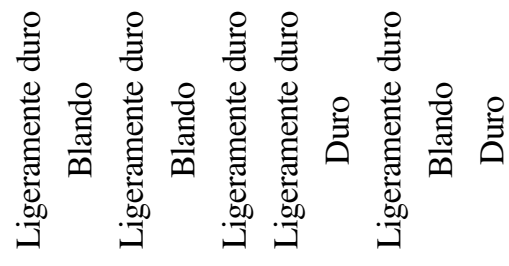 \\
\hline 苞 & 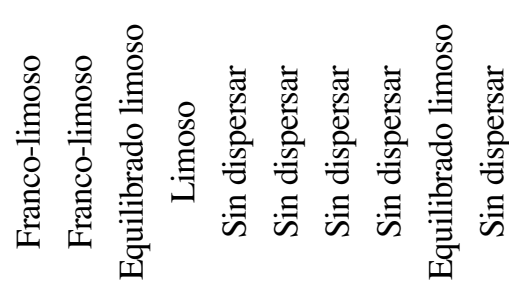 \\
\hline 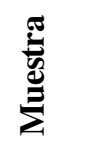 & 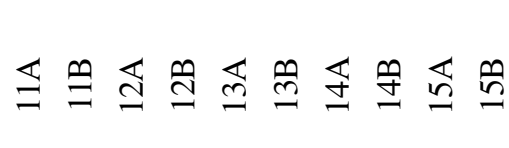 \\
\hline
\end{tabular}



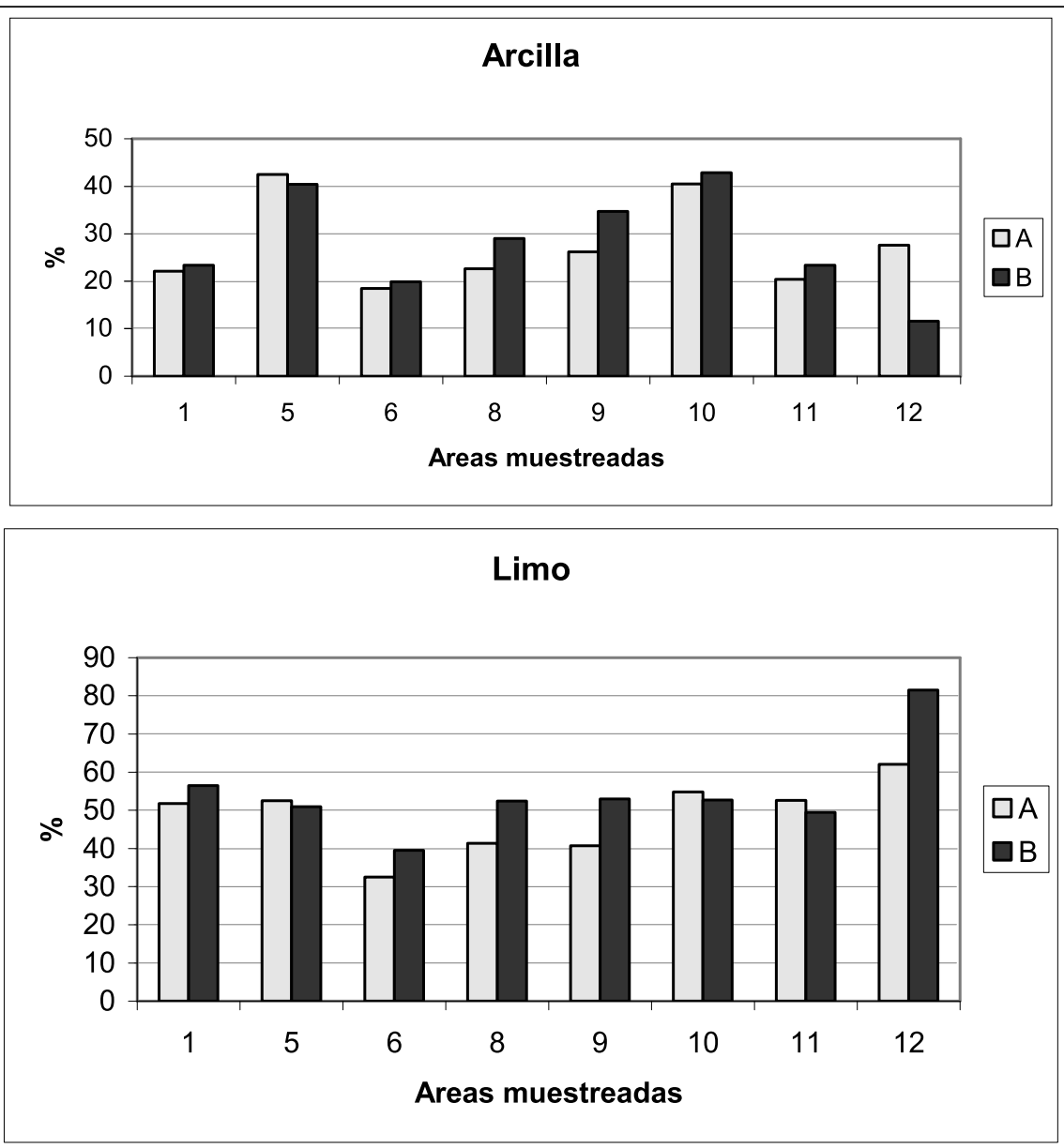

Arenas

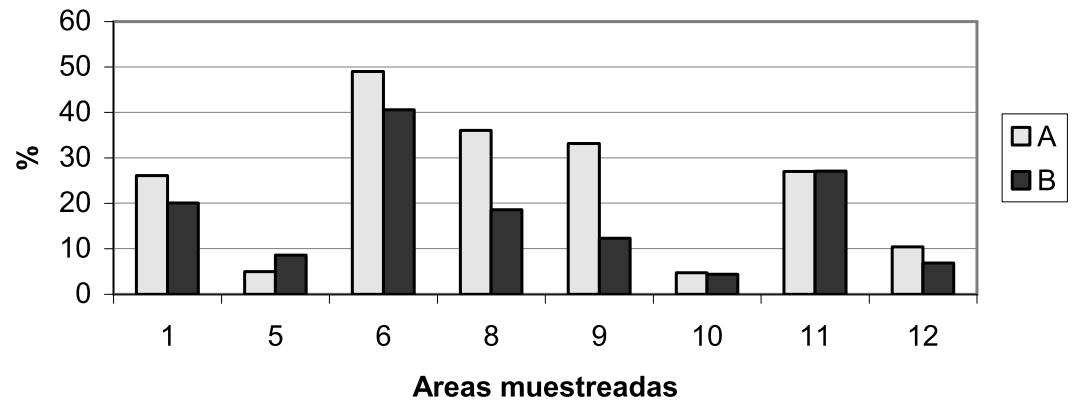

Figura 6. Porcentaje de arcilla, limo y arena de las muestras que dispersaron 
solvatación con etilénglicol pone claramente de manifiesto la gran cantidad de minerales hinchables, esmectitas. Los difractogramas de las arcilla sometidas a ataque ácido revelan que sólo existen trazas de caolinita en las muestras y que por lo tanto la reflexión a $7 \AA$ corresponde tanto a la caolita como a la clorita.

Estas arcillas hinchables, con sus cambios de volumen con las fases de desecación y humedecimiento alternantes, que se presentan tan marcadamente en la región murciana, producen la formación de un agrietamento en el suelo que favorece la circulación del agua y el drenaje subsuperficial. El contenido en esmectitas parece incrementarse ligeramente con la profundidad, lo que también pudiera contribuir al desarrollo de pipes.

Tabla 4. Estimación semicuantitativa relativa de la fracción arcilla

\begin{tabular}{|c|c|c|c|c|c|}
\hline Muestra & Esmectita & Illita & Caolinita & Clorita & Otros \\
\hline $12 \mathrm{~A}$ & +++ & +++ & $\mathrm{T}$ & + & $\mathrm{T}$ \\
$12 \mathrm{~B}$ & +++ & ++ & $\mathrm{T}$ & + & $\mathrm{T}$ \\
$13 \mathrm{~A}$ & ++ & ++++ & $\mathrm{T}$ & + & $\mathrm{T}$ \\
$13 \mathrm{~B}$ & +++ & +++ & $\mathrm{T}$ & $\mathrm{T}$ & $\mathrm{T}$ \\
$14 \mathrm{~A}$ & ++ & ++++ & $\mathrm{T}$ & + & $\mathrm{T}$ \\
$14 \mathrm{~B}$ & +++ & +++ & + & $\mathrm{T}$ & $\mathrm{T}$ \\
$15 \mathrm{~A}$ & +++ & +++ & + & $\mathrm{T}$ & $\mathrm{T}$ \\
$15 \mathrm{~B}$ & +++ & +++ & $\mathrm{T}$ & $\mathrm{T}$ & $\mathrm{T}$ \\
\hline
\end{tabular}

$\mathrm{T}=\operatorname{trazas}<5 \% ;+=5-15 \% ;++=15-30 \% ;+++=30-50 \% ;++++=50-80 \%$

\subsection{Propiedades químicas de los suelos}

Los suelos estudiados presentan unas características analíticas generales (tablas 5, 6 y 7) bastante similares, que están en consonancia con la naturaleza del material original, el ambiente climático bajo el que se encuentran y el tipo de vegetación existente.

Los valores de carbono orgánico son muy bajos en todas las muestras, con un valor medio del $0.5 \%$ y valores máximos y mínimos de 0.6 y 0.3 respectivamente. Constituyen suelos muy pobres en materia orgánica, por el escaso aporte de restos vegetales de la vegetación xerofítica muy abierta que les caracteriza. La mineralización de estos restos está muy favorecida por el clima, el elevado contenido en carbonato cálcico y por el constante rejuvenecimiento, con pérdida de materiales, a que se ven sometidos por el efecto de la erosión. Como era de esperar, los valores de carbono orgánico disminuyen en la mayoría de los casos con la profundidad, con la excepción de dos de las áreas analizadas (figura 7). La materia orgánica, evidentemente en relación con el carbono orgánico, presenta valores muy bajos también, con una media de $0.8 \%$ y un máximo de 1.2 en el área de Mazarrón. 
Tabla 5. Carbono orgánico, materia orgánica, carbonato cálcico y pH

\begin{tabular}{|c|c|c|c|c|c|}
\hline Muestras & $\begin{array}{c}\text { C.O. } \\
\%\end{array}$ & $\begin{array}{c}\text { M.O. } \\
\%\end{array}$ & $\begin{array}{c}\mathrm{CaCO}_{3} \\
(\%)\end{array}$ & $\begin{array}{c}\mathbf{p H} \\
\mathbf{H}_{2} \mathrm{O}\end{array}$ & $\begin{array}{c}\mathbf{p H} \\
\mathbf{K C l}\end{array}$ \\
\hline $1 \mathrm{~A}$ & 0,63 & 1,08 & 60,4 & 7,9 & 7,6 \\
\hline $1 \mathrm{~B}$ & 0,58 & 0,99 & 58,1 & 7,9 & 7,5 \\
\hline $2 \mathrm{~A}$ & 0,47 & 0,81 & 36,0 & 7,7 & 7,5 \\
\hline $2 \mathrm{~B}$ & 0,43 & 0,74 & 35,4 & 7,8 & 7,5 \\
\hline $3 \mathrm{~A}$ & 0,37 & 0,64 & 46,9 & 7,8 & 7,5 \\
\hline $3 B$ & 0,40 & 0,69 & 47,8 & 8,7 & 8,0 \\
\hline $4 \mathrm{~A}$ & 0,50 & 0,86 & 54,5 & 8,0 & 7,6 \\
\hline $4 B$ & 0,46 & 0,79 & 51,7 & 8,1 & 7,6 \\
\hline $5 \mathrm{~A}$ & 0,48 & 0,82 & 56,4 & 7,9 & 7,6 \\
\hline $5 B$ & 0,33 & 0,57 & 54,4 & 7,9 & 7,5 \\
\hline $6 \mathrm{~A}$ & 0,50 & 0,86 & 61,8 & 8,4 & 7,7 \\
\hline $6 \mathrm{~B}$ & 0,55 & 0,95 & 60,9 & 8,4 & 7,6 \\
\hline $7 \mathrm{~A}$ & 0,59 & 1,05 & 48,5 & 8,2 & 7,4 \\
\hline $7 \mathrm{~B}$ & 0,49 & 0,84 & 37,8 & 7,9 & 7,4 \\
\hline $8 \mathrm{~A}$ & 0,55 & 0,95 & 64,4 & 8,4 & 7,5 \\
\hline $8 B$ & 0,54 & 0,93 & 60,3 & 8,2 & 7,4 \\
\hline $9 \mathrm{~A}$ & 0,33 & 0,57 & 89,0 & 8,4 & 7,5 \\
\hline $9 B$ & 0,29 & 0,50 & 80,3 & 8,2 & 7,6 \\
\hline $10 \mathrm{~A}$ & 0,54 & 0,93 & 56,4 & 8,4 & 7,6 \\
\hline $10 \mathrm{~B}$ & 0,60 & 1,03 & 54,0 & 7,9 & 7,4 \\
\hline $11 \mathrm{~A}$ & 0,70 & 1,20 & 50,2 & 8,2 & 7,7 \\
\hline $11 \mathrm{~B}$ & 0,52 & 0,89 & 56,4 & 7,9 & 7,6 \\
\hline $12 \mathrm{~A}$ & 0,47 & 0,81 & 66.6 & 8.0 & 7.6 \\
\hline $12 \mathrm{~B}$ & 0,38 & 0,65 & 57.4 & 8.0 & 7.6 \\
\hline $13 \mathrm{~A}$ & 0,50 & 0,86 & 59.6 & 8.0 & 7.5 \\
\hline $13 \mathrm{~B}$ & 0,29 & 0,50 & 57.0 & 8.2 & 7.6 \\
\hline $14 \mathrm{~A}$ & 0,44 & 0,76 & 59.9 & 8.1 & 7.4 \\
\hline $14 \mathrm{~B}$ & 0,30 & 0,52 & 66.8 & 8.1 & 7.7 \\
\hline $15 \mathrm{~A}$ & 0,60 & 1,03 & 63.3 & 8.0 & 7.6 \\
\hline $15 \mathrm{~B}$ & 0,41 & 0,70 & 60.3 & 8.0 & 7.6 \\
\hline
\end{tabular}


Tabla 6. Cationes asimilables y capacidad de cambio catiónico

\begin{tabular}{|c|c|c|c|c|c|}
\hline Muestras & $\begin{array}{c}\mathrm{Na} \\
\mathrm{mg} / \mathbf{1 0 0 g}\end{array}$ & $\underset{\mathrm{mg} / 100 \mathrm{~g}}{\mathrm{~K}}$ & $\begin{array}{c}\mathrm{Mg} \\
\mathrm{mg} / 100 \mathrm{~g}\end{array}$ & $\begin{array}{c}\text { C.C.C. } \\
\text { cmol }_{\mathrm{cg}^{-1}}\end{array}$ & $\begin{array}{c}\text { P.S.C. } \\
\%\end{array}$ \\
\hline $1 \mathrm{~A}$ & 17,50 & 13,75 & 28,75 & 10,34 & 7,35 \\
\hline 1B & 26,25 & 13,75 & 33,75 & 10,56 & 10,79 \\
\hline $2 \mathrm{~A}$ & 11,25 & 27,00 & 35,00 & 11,88 & 4,12 \\
\hline $2 B$ & 19,00 & 15,50 & 25,00 & 11,66 & 7,03 \\
\hline $3 \mathrm{~A}$ & 36,50 & 35,75 & 73,75 & 13,20 & 11,9 \\
\hline $3 B$ & 262,50 & 35,25 & 66,25 & 13,42 & 85,11 \\
\hline $4 \mathrm{~A}$ & 175,00 & 55,25 & 118,75 & 11,66 & 65,18 \\
\hline $4 B$ & 150,00 & 54,25 & 88,75 & 13,64 & 47,80 \\
\hline $5 \mathrm{~A}$ & 32,75 & 30,50 & 70,00 & 13,42 & 10,60 \\
\hline $5 B$ & 50,00 & 30,50 & 72,50 & 11,44 & 18,97 \\
\hline $6 \mathrm{~A}$ & 15,75 & 17,75 & 17,50 & 5,50 & 12,36 \\
\hline $6 \mathrm{~B}$ & 13,75 & 10,00 & 21,25 & 7,04 & 8,40 \\
\hline $7 \mathrm{~A}$ & 13,50 & 25,50 & 26,25 & 11,00 & 5,27 \\
\hline $7 \mathrm{~B}$ & 15,25 & 26,25 & 36,25 & 9,24 & 7,14 \\
\hline $8 \mathrm{~A}$ & 14,50 & 16,50 & 28,75 & 9,68 & 6,50 \\
\hline $8 B$ & 17,00 & 14,75 & 45,00 & 12,78 & 5,79 \\
\hline $9 \mathrm{~A}$ & 34,25 & 7,50 & 30,00 & 4,84 & 30,78 \\
\hline $9 \mathrm{~B}$ & 103,50 & 24,00 & 77,50 & 11,66 & 38,60 \\
\hline $10 \mathrm{~A}$ & 15,75 & 31,50 & 48,75 & 14,08 & 4,83 \\
\hline 10B & 94,00 & 28,00 & 61,25 & 13,64 & 29,91 \\
\hline $11 \mathrm{~A}$ & 18,25 & 42,00 & 18,75 & 9,24 & 1,46 \\
\hline $11 \mathrm{~B}$ & 21,75 & 12,50 & 38,75 & 10,34 & 9,78 \\
\hline $12 \mathrm{~A}$ & 6,00 & 16,50 & 20,00 & 17,78 & 1,31 \\
\hline $12 B$ & 40,00 & 16,25 & 40,00 & 17,78 & 10,76 \\
\hline $13 \mathrm{~A}$ & 5,50 & 14,25 & 50,00 & 18,28 & 8,19 \\
\hline $13 \mathrm{~B}$ & 44,00 & 12,25 & 52,50 & 17,78 & 7,96 \\
\hline $14 \mathrm{~A}$ & 39,25 & 17,00 & 52,50 & 20,82 & 1,48 \\
\hline $14 \mathrm{~B}$ & 40,00 & 12,50 & 30,00 & 21,84 & 9,27 \\
\hline $15 \mathrm{~A}$ & 5,00 & 15,00 & 22,50 & 14,73 & 1,46 \\
\hline $15 B$ & 32,50 & 12,50 & 62,50 & 15,24 & 9,78 \\
\hline
\end{tabular}




\begin{tabular}{|c|c|}
\hline$\dot{\vec{\theta}}$ & 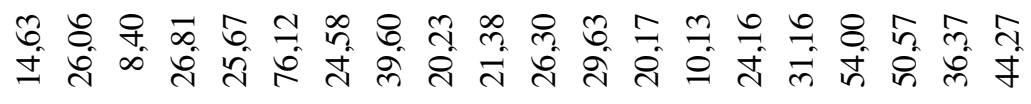 \\
\hline 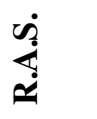 & 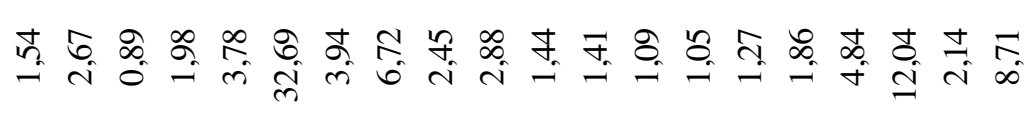 \\
\hline ن & 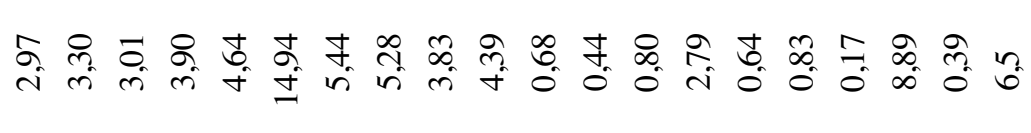 \\
\hline " & 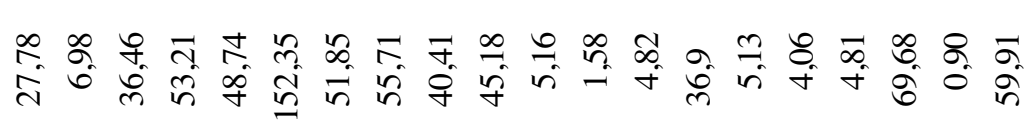 \\
\hline 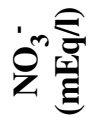 & 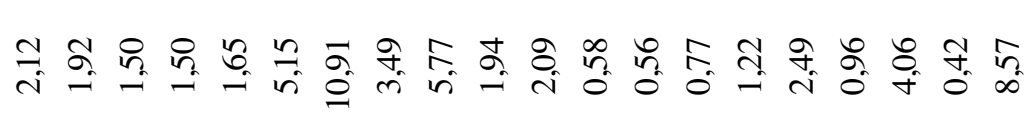 \\
\hline 它 & 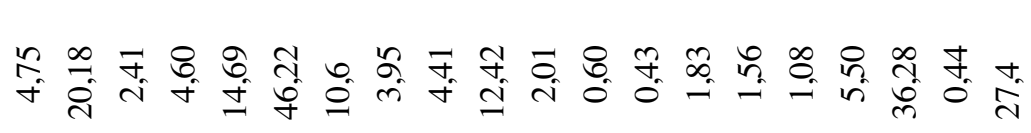 \\
\hline 䒫 & 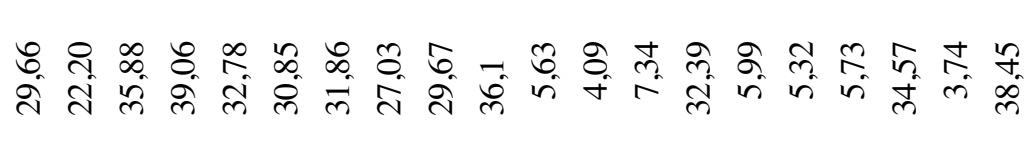 \\
\hline 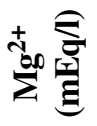 & 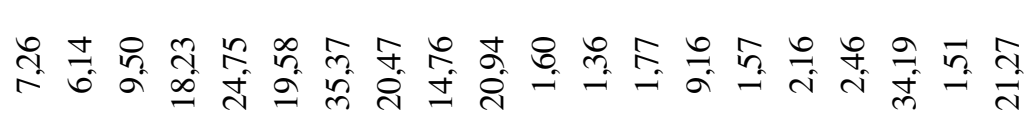 \\
\hline 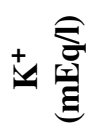 & 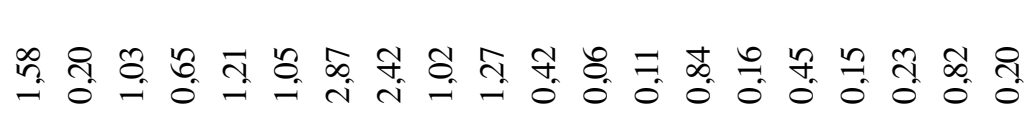 \\
\hline 旅 & 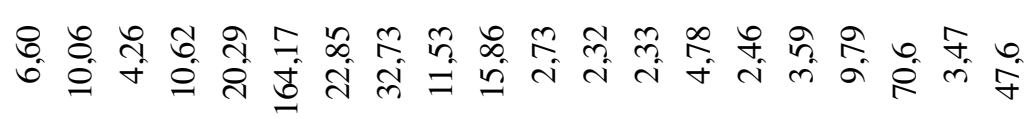 \\
\hline 焉 & 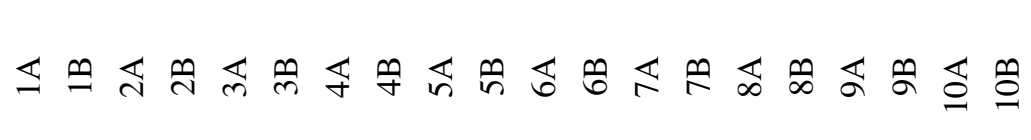 \\
\hline
\end{tabular}




\begin{tabular}{|c|c|}
\hline $\begin{array}{l}\dot{0} \\
\dot{0} \\
\dot{n}\end{array}$ & 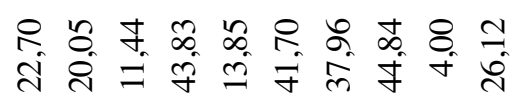 \\
\hline 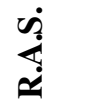 & 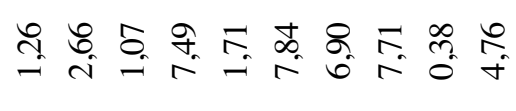 \\
\hline نَّ & 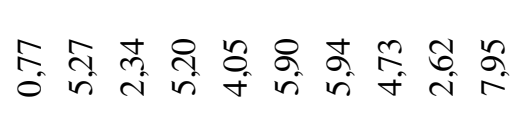 \\
\hline " & 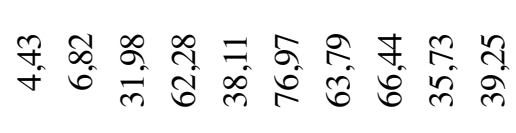 \\
\hline 的窟 & 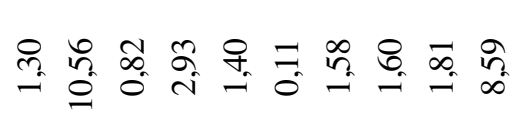 \\
\hline 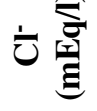 & 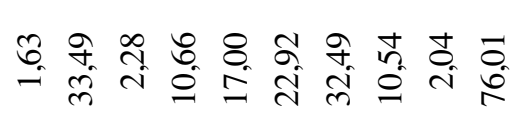 \\
\hline 志 & 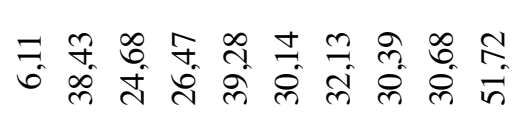 \\
\hline 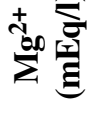 & 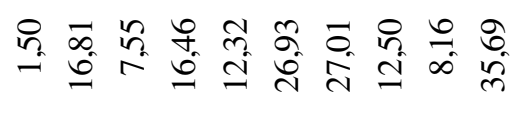 \\
\hline 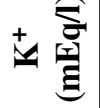 & 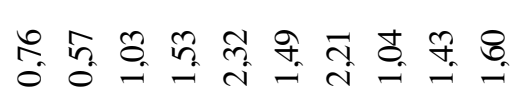 \\
\hline 槖离 & 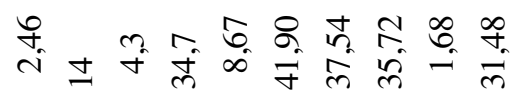 \\
\hline 窇 & 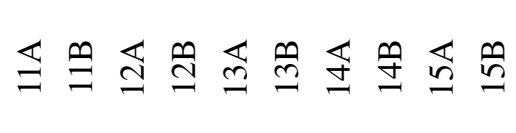 \\
\hline
\end{tabular}


Son suelos muy calizos, con un valor medio superior al 50\%. Los niveles superficiales oscilan entre 36 y $89 \%$ y los más profundos entre 35 y $80 \%$. Por lo general los valores en superficie son mas elevados que en profundidad, con la excepción de las áreas 11 y 14 de muestreo (figura 8). El área 11, se corresponde con el área de Mazarrón donde junto a las margas hay una importante presencia de conglomerados; y el área 14 constituye una zona aterrazada en los años 80 del siglo pasado, pero que nunca se llegó a poner en cultivo, por lo que la mezcla de horizontes edáficos y su no cultivo puede explicar las anomalías encontradas en varios de los parámetros analizados.

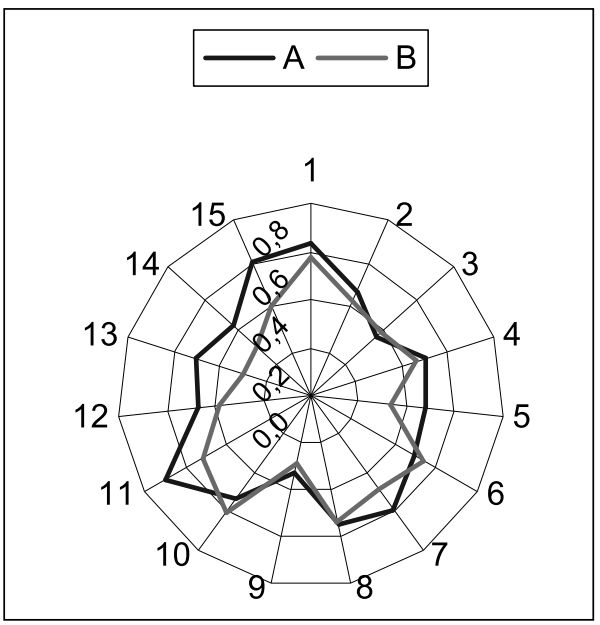

Figura 7. Carbono orgánico (\%) ( $A=$ Superficie $; B=$ Profundidad $)$

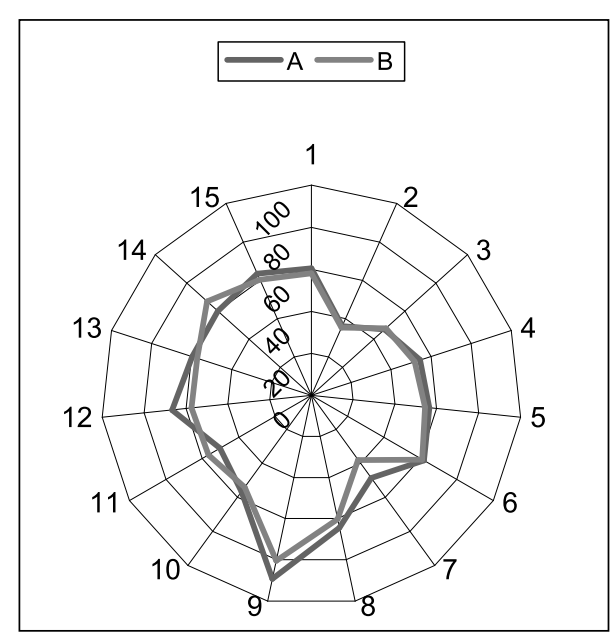

Figura 8. Carbonato cálcico (\%)

En consonancia con el alto contenido en carbonato cálcico de estos suelos, sus valores de $\mathrm{pH}$ son elevados, alcalinos y muy próximos entre sí; los pHs determinados en agua y en solución de cloruro potásico $1 \mathrm{~N}$, lo que indica la total saturación del complejo de cambio en bases y el predominio del calcio entre los cationes de cambio. De todas formas el valor más elevado es de 8.7 , valores superiores indicarían problemas de alcalinidad.

Para conocer la salinidad de estos suelos se midió la conductividad eléctrica del extracto de saturación (tabla 7). A excepción de dos áreas, la número 6 localizada en Abanilla y la 8 en Calasparra, superan el umbral crítico de $2 \mathrm{dS} \mathrm{m}^{-1}$ a partir del cual se considera salinidad en el suelo; por tanto se puede considerar que estos suelos están afectados por sales. Los valores de salinidad aumentan en profundidad en todos los casos (figura 10), salvo en el caso del área 14. Este aumento de la salinidad, y de manera muy importante en algunos áreas, incrementa el efecto de sifón a estos niveles. Esto podría justificar el comienzo del proceso de piping en los niveles subsuperficiales. En el caso de las sales más solubles que el yeso, su acción es mucho más notable, ya que, al aumentar la presión osmótica de la solución de suelos, origina suelos fisiológicamente secos, incluso para bajas concen- 


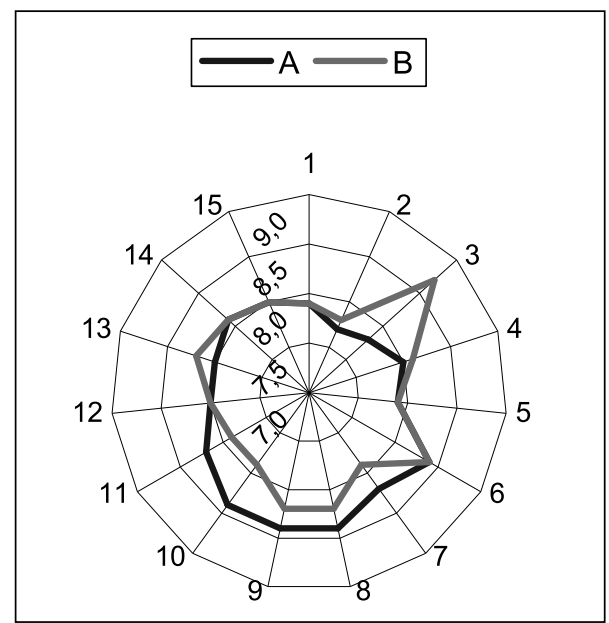

Figura 9. $\mathrm{PH}_{2} \mathrm{O}$ de las áreas muestreadas

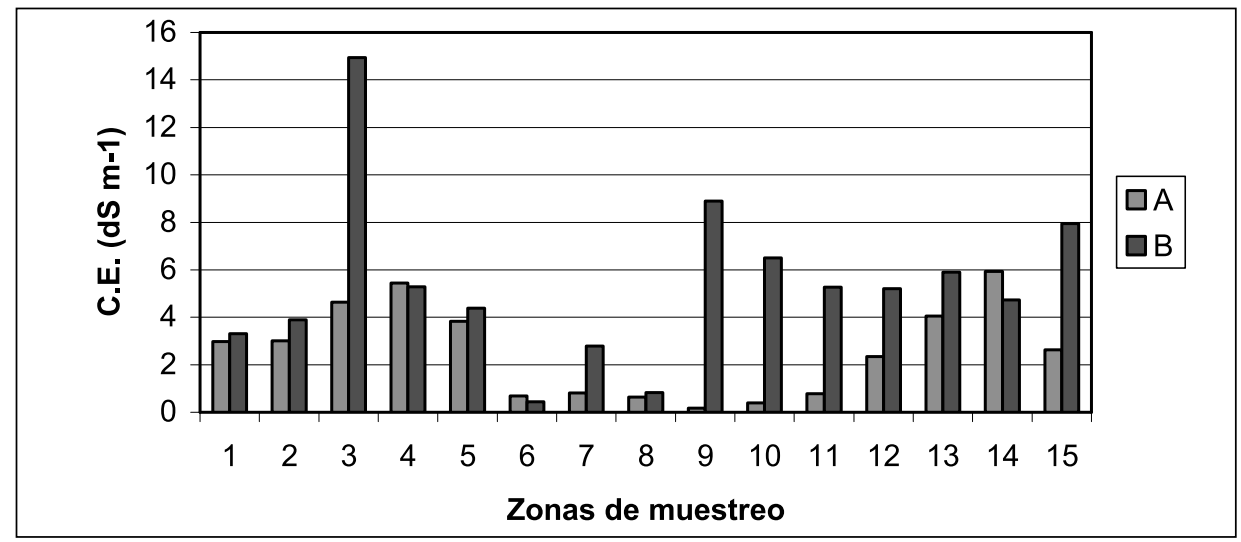

Figura 10. Conductividad eléctrica

traciones salinas. Tan sólo en el área 14 (comentada anteriormente, aterrazada pero no cultivada), los valores de salinidad son más elevados en superficie, tal vez debido a que fue el único suelo que no se cultivó años atrás, mientras que los otros suelos si que se cultivaron y el arado superficial facilitó el lavado de las sales con las aguas de infiltración.

Los constituyentes del suelo responsables de su capacidad de cambio son fundamentalmente la materia orgánica y la fracción arcilla, que influye por su cantidad pero también por su mineralogía, siendo las de tipo 2:1 las que tienen mayor capacidad de cambio. Los valores que presentan estos suelos son altos (tabla 6) debido fundamentalmente a la cantidad y naturaleza de su fracción arcilla, constituída por illitas y esmectitas preferentemente (Sánchez, 1975). Estas arcillas hinchables, con sus cambios de volu- 
men con las fases de desecación y humedecimiento alternantes, que se presentan tan marcadamente en la región murciana, producen la formación de un agrietamento en el suelo que favorece la circulación del agua y el drenaje subsuperficial.

Los niveles de sodio, potasio y magnesio asimilables, que incluyen sus valores solubles y los cambiables, absorbidos en el complejo de cambio, se recogen también en la tabla 6. Posteriormente, se ha calculado el porcentaje de sodio de cambio (P.S.C.), presentando generalmente valores más altos en profundidad que en superficie (figura 11). En el $50 \%$ de las áreas, el sodio asimilable es cinco o seis veces más elevado a $100 \mathrm{~cm}$ de profundidad que en superficie (figura 12), esto justifica lo que ya se esperaba, y apunta de nuevo a que la presencia de sodio en el complejo de cambio facilita la dispersión de las arcillas (Heede, 1971) y así el transporte de materiales sólidos en suspensión.

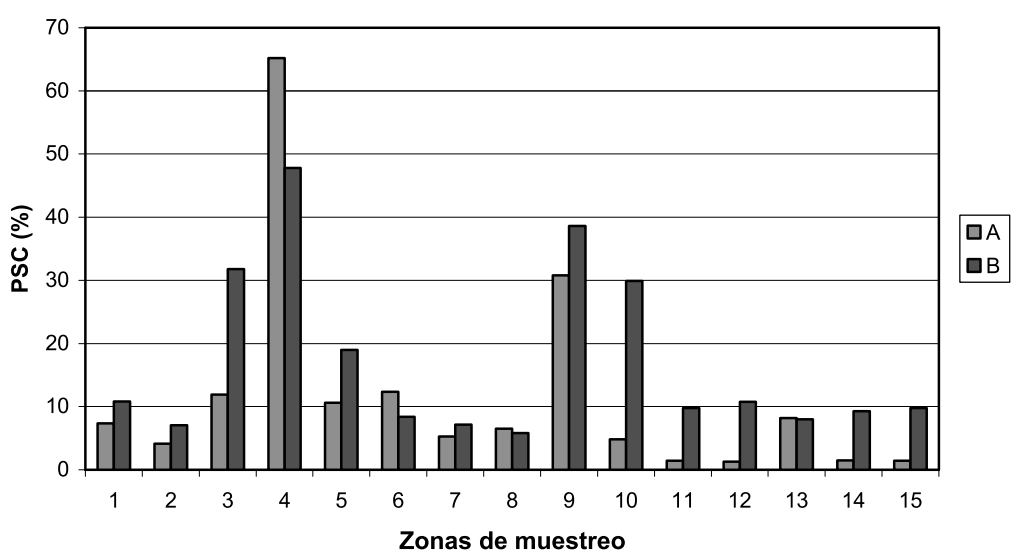

Figura 11. Porcentaje de Sodio de Cambio

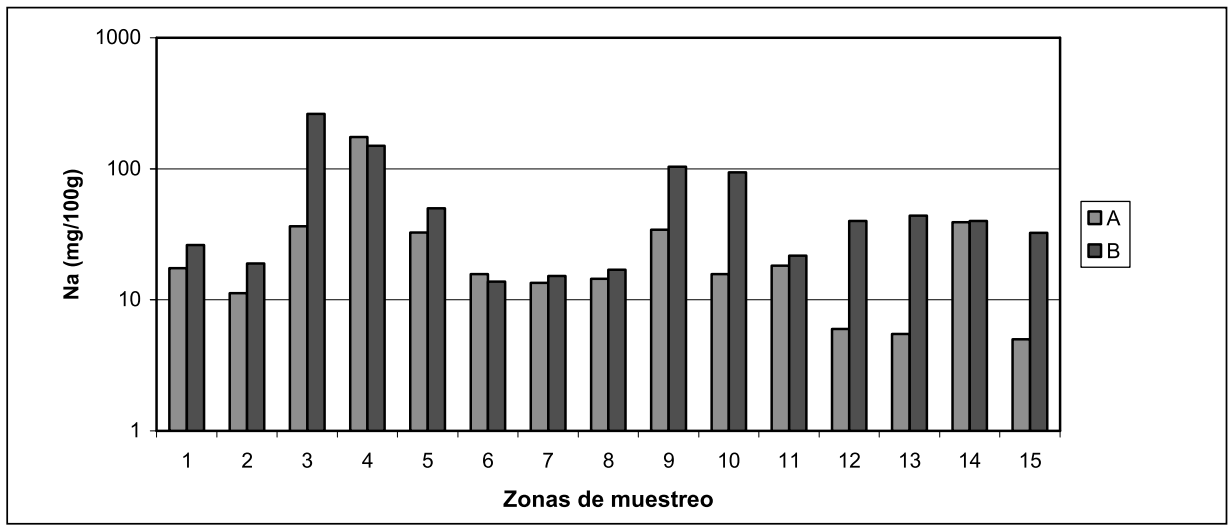

Figura 12. Sodio asimilable 
Es de destacar nuevamente el área 14, donde tanto en superficie como en profundidad, el sodio asimilable presenta valores muy igualados, explicados por el manejo distinto que se hizo en el pasado de estos suelos al ser aterrazados pero no cultivados; ello hace que hayan quedado más expuesto a estos procesos que las otras zonas que sí se cultivaron y en la que se observan valores de sodio mucho más bajos en superficie, justo en la profundidad de capa arable que estuvo afectada por las labores de cultivo.

Los niveles de potasio se mantienen casi constantes, sólo ligeramente más altos en superficie (tabla 6), tal vez por el aporte de fertilizantes en el pasado o por la influencia de la materia orgánica.

La composición salina del extracto de saturación muestra en todos sus elementos, a excepción del potasio, valores medios mucho más elevados en profundidad que en superficie (tabla 7 y figura 13), en especial el sodio y los sulfatos. La abundancia de sulfatos se justifica por ser muestras muy yesíferas.

Con el objeto de observar las diferencias entre los niveles superficiales y los más profundos en cuanto a salinidad se han establecido relaciones entre la Razón de Adsorción de Sodio (R.A.S) y la Conductividad Eléctrica (C.E.) de las diferentes muestras en conjunto y por separado, en superficie y a $100 \mathrm{~cm}$ de profundidad. Los resultados muestran una muy buena relación de las muestras en profundidad y escasa relación en las de superficie (figura 14). Este hecho, de nuevo, pone de manifiesto las características favorables de los niveles profundos para el desarrollo de los procesos de piping.

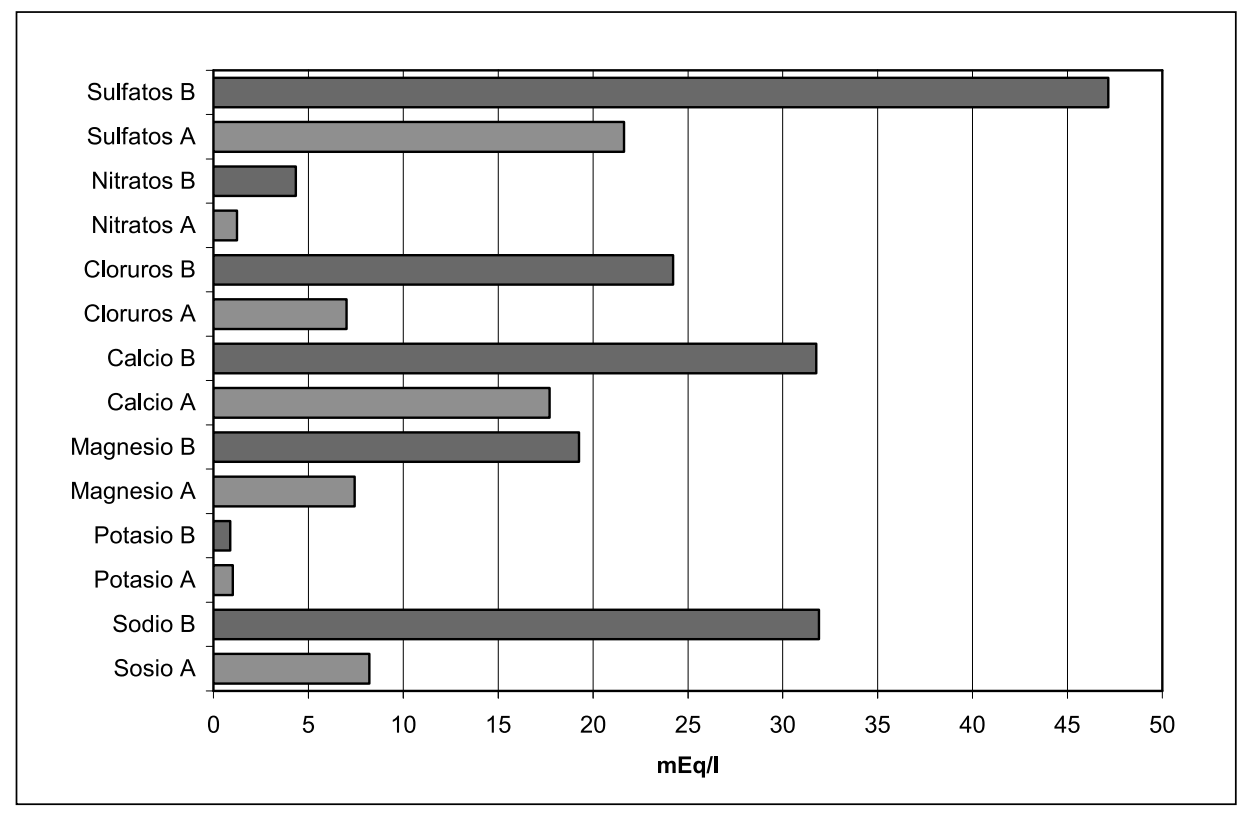

Figura 13. Valores medios de la composición salina del extracto de saturación 


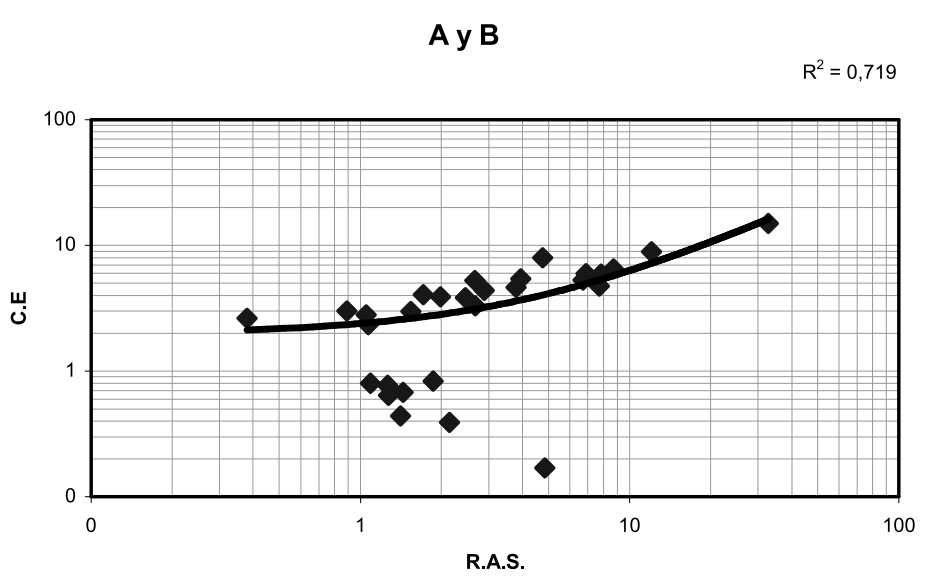

A

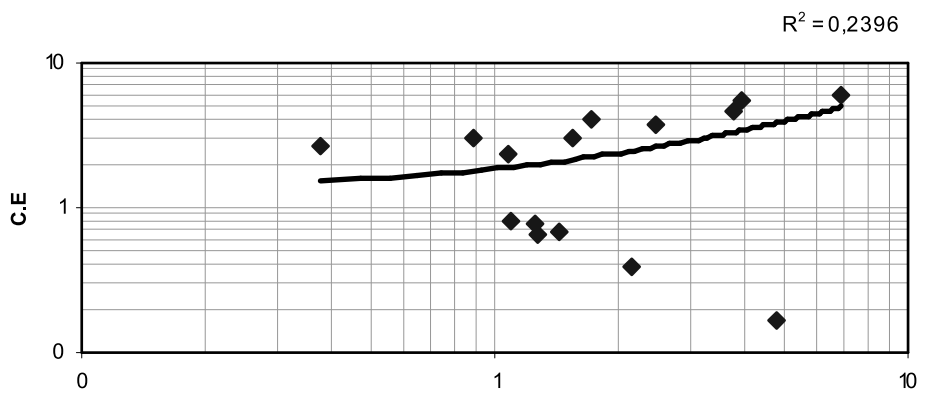

R.A.S.

B

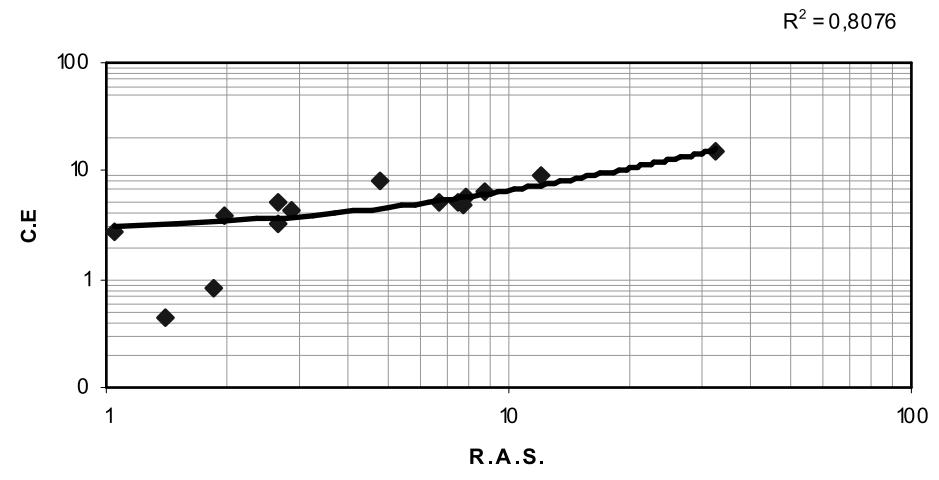

Figura 14. Relación entre R.A.S. y C.E. a distintas profundidades 


\section{Conclusiones}

Después del estudio realizado se puede afirmar que la degradación de las tierras afectadas por piping en la Región de Murcia es realmente importante. Las 67 áreas inventariadas y otras muchas existentes, de menores dimensiones, son evidencia de ello.

Las áreas preferentes para el desarrollo de piping son áreas margosas, con variaciones en sus características físicas y químicas, tanto en superficie como en profundidad, aterrazadas para su cultivo y con posterioridad abandonadas. Las formas de erosión, en ocasiones, son tan acentuadas que es imposible recuperar estas tierras para el cultivo.

El proceso de piping aparece ligado, predominantemente, a áreas aterrazadas de escasa pendiente y con importantes gradientes hidráulicos, generados por la altura entre dos terrazas de cultivo. La altura entre parcelas condiciona la profundidad que alcanzan los pipes. Por otro lado, la escasa pendiente de las terrazas favorece el estancamiento y la infiltración de agua, en lugar de la escorrentía, provocando la formación de conductos subsuperficiales.

Algunas de las propiedades físicas de los suelos, aquí estudiados, favorecen la aparición y desarrollo del piping, como las diferencias de estructura y textura a diferentes profundidades. En superficie, los suelos están pobremente estructurados, pero en profundidad carecen de ella. Respecto a la textura es más fina en profundidad que en superficie, siendo limo-arcillosa en los primeros centímetros del suelo y arcillo-limosa en las capas mas profundas. Es de destacar también la presencia de arcillas expansivas, responsables de la aparición de grietas, que favorecen el inicio del proceso de piping.

Respecto a las propiedades químicas destacables de estos suelos, en relación con la formación de piping, está el alto contenido en sales muy solubles y carbonato cálcico. De gran importancia es el elevado porcentaje de sodio de cambio, en especial en los niveles mas profundos, lo cual favorece la dispersión de la arcilla y la aparición del proceso de piping en los niveles subsuperficiales.

Como conclusión general se podría decir que los campos de cultivo aterrazados, que han sido posteriormente abandonados, sobre litologías de textura fina (limo-arcillosas y arcillo-limosas) y con diferencias a distintas profundidades, con altos contenidos en sales, escasa cobertura vegetal, en condiciones semiáridas y con importantes gradientes entre las distintas terrazas de cultivo constituyen un espacio potencialmente óptimo para el desarrollo de los procesos de piping. Las áreas con estas características en la Región de Murcia son abundantes, por lo que es importante hacer una valoración de los efectos que estos procesos de erosión ocasionan, no solo por la pérdida de superficies de tierras de cultivo, sino también por las elevadas pérdidas de suelos que en estos lugares se producen. Las altas tasas de erosión, producto de los procesos de piping, consecuentemente, llevan ligadas la acumulación posterior de estos sedimentos en diferentes obras de infraestructura, ocasionando así su aterramiento y pérdida de funcionalidad. 
Una práctica de conservación de suelo habitual en el área mediterránea como es el aterrazamiento, en áreas como las que aquí se han estudiado, ocasiona el efecto contrario. Las terrazas crean el gradiente hidráulico necesario para el desarrollo de los procesos de piping y cuanto más altas son las terrazas construidas, mayor es el grado de erosión que se puede dar en estas terrazas, tras su abandono.

\section{Referencias bibliográficas}

Arana Castillo, R., Rodríguez Estrella, T., Mancheño Jiménez, M.A., Ortiz SILlA, R. (1992). Lugares de interés geológico de la Región de Murcia. Agencia para el Medio Ambiente y la Naturaleza. Serie Técnica 1, 219 pp. Murcia.

Barrón, G., Echeverría, M. T., Ibarra, P., Marco, P., Pérez, F. (1994). Algunas consecuencias geomorfológicas del uso del suelo agrícola en las últimas décadas. La actividad del piping en el bajo valle del Huerva (Zaragoza, Spain). En: Geomorfología en España, Tomo II (Arnáez, J., García Ruiz, J.M., Gómez Villar, A., Eds.). Sociedad Española de Geomorfología, 255-266 pp.

Bower, C.A., WiLcox, L.V. (1965). Soluble salts. In: Methods of Soil Analysis, part 2 (Black, C.A., Ed.). Amer. Soc. Agronomy, Inc. Madison. Wisconsin. EEUU. 933$940 \mathrm{pp}$.

BRYAN, R., JONES, J. A. A. (1997). The significance of soil piping processes: inventory and prospect. Geomorphology, 20: 209-218.

Calvo Cases, A., Harvey, A. M., Paya-Serrano, J. (1991). Process interactions and badland development in SE Spain. In: Soil Erosion Studies in Spain (Sala, M., Rubio, J.L., García Ruíz, J.M., Eds). Geoforma Ediciones, 75-90 pp. Logroño.

Calvo Cases, A., Harvey, A. M. (1996). Morphology and development of selected badlands in S.E. Spain: implications of climatic change. Earth Surface Processes and Landforms. 21: 725-735.

Chapman, H.D. (1965). Cation exchange capacity. In: Methods of Soil Analysis, part 2 (Black, C.A., Ed.). Amer. Soc. Agronomy, Inc. Madison. Wisconsin. EEUU. 891-900 pp.

CERDÀ, A. (1998). Soil aggregate stability under different Mediterranean vegetation types. Catena. 32: 73-86.

Del Valle, J. M., Val Del, J. (1990). Procesos de erosión y análisis de sus condicionantes en una región semiárida: La cuenca de Cornialto (Bárdenas, Navarra). Cuaternario y Geomorfología, 4: 55-67.

Duchaufour, P.H. (1970). Pedalogie. Masson. París. 
FAO, (1977). Guidelines for soil description. FAO-ISRIC. Roma.

FAO-ISRIC-ISSS, (1999). World Reference Base for Soil Resources. Atlas. Acco, Leuven. Bélgica.

FAUlKNER, H., SPIVEY, D., AlEXANDER, R. (2000). The role of some site geochemical processes in the development and stabilisation of three badland sites in Almería, southern Spain. Geomorphology. 35: 87-99.

FAUlKner, H., AleXANDER, R., Wilson, B. R. (2003). Changes to the dispersive characteristics of soils along an evolutionary slope sequence in the Vera badlands, southeast Spain: implications for site stabilisation. Catena. 50: 243-254.

FAUlKNER, H., RuIZ, J., ZuKOWSKYJ, P., DOWNwARD, S. (2003). Erosion risk associated with rapid and extensive agricultural clearances on dispersive materials in southeast Spain. Environmental Science \& Policy. 6: 115-127.

FAUlKNER, H. (2006). Piping hazard on collapsible and dispersive soils in Europe. In: Soil Erosion in Europe (Boardman, J. \& Poesen, J., Eds.). John Wiley \& Sons, Ltd., 537- $562 \mathrm{pp}$.

Ferre Bueno, E., Asensi Marfil, A., Senciales GonzÁlez, J.M. (1994). Procesos de erosión y dinámica de la vegetación en bancales abandonados en el valle del Andarax (provincia de Almería). En: Efectos geomorfológicos del abandono de tierras (García Ruiz, J.M., Lasanta, T., Eds.). Sociedad Española de Geomorfología, 31-41 pp. Zaragoza.

GALLART, F. (1979). Observaciones sobre la geomorfología dinámica actual en la Conca d' Odena (alrededores de Igualada, provincia de Barcelona). Actas de la III Reunión Nacional del Grupo Español de Trabajo del Cuaternario, 123-134 pp.

García Ruiz, J. M., Lasanta, T., Ortigosa IzQuierdo, Arnáez VAdillo, J. (1986). Pipes in cultivated soils of La Rioja: Origin and evolution. Z. Geomorph, Suppl-Bd,. 58: 93-100.

García Ruiz, J. M., Lasanta, T., Alberto, F. (1994). Pérdida de sedimentos por sufusión en campos de regadío. En: Geomorfología en España. Tomo II (Arnáez, J., García Ruiz, J.M., Gómez Villar, A., Ed.). Sociedad Española de Geomorfología, 267276 pp. Logroño.

García Ruiz, J. M., LASANTA, T., AlBerTO, F. (1997). Soil erosion by piping in irrigated fields. Geomorphology, 20 (3-4): 269-278.

GerZABEK, M.H., KiRCHMAN, PiChlMAYER, F. (1995). Response of soil aggregate stability to manure amendments in Ultuma long-term organic matter experiment. Zeitschrift für Pflanzenernährung und Bodenkunde, 158 (3), 257-560. 
Gracia PRIETO, F. J. (1986). Dinámica erosiva del piping: Un ejemplo en la Depresión del Ebro. Cuadernos de Investigación Geográfica, 12: 11-24.

GutIÉRREZ, M., RODRÍGUEZ, J. (1984). Fenómenos de sufusión (piping) en la depresión media del Ebro. Cuadernos de Investigación Geográfica, 10: 75-83.

GutiÉrRez, M., BEnito, G., RodríGueZ, J. (1988). Piping in badland areas of the middle Ebro basin, Spain. Catena Suppl. 13: 49-60.

Gutiérrez, M., SAncho, C., Benito, G., Sirvent, J., Desir, G. (1994). Procesos generadores de piping en zonas de badlands de la Depresión del Ebro. En: Geomorfología en España. Tomo II (Arnáez, J., García Ruiz, J.M., Gómez Villar, A., Ed.). Sociedad Española de Geomorfología, 277-285 pp. Logroño.

Gutiérrez, M., Sancho, C., Benito, G., Sirvent, J., Desir, G. (1997). Quantitative study of piping processes in badland areas of the Ebro Basin, NE Spain. Geomorphology, 20 (3-4): 237-253.

HARVEY, A. (1982). The role of piping in the development of badlands and gully systems in south-east Spain. In: Badland. Geomorphology and Piping (Bryan, R. \& Yair, A., Eds.) Geobooks. Cambridge, 317-335 pp.

HEEDE, B.H. (1971). Characteristics and processes of soil piping in gullies. United States Department of Agriculture Forest Service, Rocky Mountain Forest and Range. Experiment Station Research Paper RM-68. 15 pp

HiLlel, D. (1980). Soil Structure and Aggregation. In: Fundamentals of Soil Physics. Academic Press, Inc. 93-119 pp. New York.

JONES, J. A. A. (1981). The nature of soil piping a review of research. Research Monograph $\mathrm{n}^{\circ} .3, \mathrm{BGRG}$, Geobooks, Norwich, $301 \mathrm{pp}$.

LaX, A., Díaz, E., Castillo, V., Albaladejo, J. (1994). Reclamation of physical and chemical properties of a salinized soil by organic amendment. Arid Soil Res. Rehab, 8: 9-17.

LóPez Bermúdez, F., Torcal, L. (1986). Procesos de erosión en túnel (piping) en cuencas sedimentarias de Murcia. Estudio preliminar mediante difracción de rayos X y microscopio de barrido. Papeles de Geografía, 11: 7-20.

LÓPEZ BERMúdez, F., Romero DíAZ, A. (1989). Arid and semi-arid environments. Geomorphological and Pedological Aspects. Catena Suppl., 14: 59-73.

Marín SANLEAndro, P., SÁnchez Soriano, A., Romero DíAz, A. (2004). The piping process in Murcia (S.E. Spain): II Effect of chemical soil properties. In: Fourth International Conference on Land Degradation. Cartagena, Spain (Faz, A., Ortiz, R., \& García, G., Ed.), Topic 6 (20). Cd Extended abstracts. 
Martín Penela, A. J. (1990). Procesos de piping en la cuenca del río Almanzora (Provincia de Almería). I Reunión Nacional de Geomorfología. 299-308 pp. Teruel.

Martín Penela, A. J. (1994). Pipe ans gully systems development in the Almanzora Basin (Southeast Spain). Z. Geomorph. N.F. 38: 207-222.

Martín Pozas, J.M., Martín Vivaldi, J.L., RodríGuez Gallego, M. (1969): Análisis cuantitativo de los filosilicatos de la arcilla por difracción de rayos X. Real Soc. Esp. Fis y Quím., Serie B.L.V: 109-112.

Mensua, S., IBAÑEZ, M.J. (1975). Alvéolos en la Depresión del Ebro. Cuadernos de Investigación. Geografia e Historia, 2: 3-14.

PARKER, C.G. (1964). Piping, a geomorphic agent in landform development of the drylands. In: Land Erosion, Precipitation, Hydrometry, Soil Moisture. Proceedings of the General Assembly of Berkeley, 19-31 August 1963. International Association of Scientific Hydrology, 65: 103-113.

PeEch, M. (1965). Hidrogen-ion activity. In: Methods of Soil Analysis, part 2 (Black, C.A., Ed.). Amer. Soc. Agronomy, Inc. Madison. Wisconsin. EEUU, 914-916 pp.

Poesen, J., Vanderkerckhole, L., Nachtergaele, J., Oostwoud Wijdenes, D., Verstraeten, G., VAN Wesemael, B. (2002). Gully erosion in Dryland Environments. In: Dryland Rivers. Hydrology and Geomorphology of Semi-Arid Channels (Bull. L.J., Kirkby, M.J., Eds.), Wiley, 229-262 pp.

RoBISON, D.A., PHILliPS, C.P. (2001). Crust development in relation to vegetation and agricultural practice on erosion susceptible, dispersive clay soils from central and southern Italy. Soil and Tillage Research, 60: 1-9.

RoDRíGueZ VidAL, J. (1982). Distribución morfoclimática en la Depresión media del Ebro: procesos dominantes y modelado actual. Estudios Geológicos, 38: 43-50.

Romero DíAZ, A., LÓPEZ BermúdeZ, F. (1985). Procesos de erosión en Cuencas Neógenas-Cuaternarias: La Cuenca de Mula. Guía de Itinerarios Geográficos de la Región de Murcia. Departamento de Geografía, Universidad de Murcia., 83-97 pp.

ROMERo DíAZ, A. (2002). Abandonment of agricultural lands and influence on erosion processes in the Region of Murcia (SE Spain). In: Sustainable Use and Management of Soils in Arid and Semiarid regions (Faz, A., Ortíz, R., Mermut, A.R., Eds.). SUMASS, 263-264 pp. Cartagena.

Romero Díaz, A., Marín SANLEANdro, P., SÁnchez Soriano, A., Belmonte SERrato, F. Y FAULKNER, H. (2007). The causes of piping in a set of abandoned agricultural terraces in southeast Spain. Catena, 69: 282-293.

SÁNCHez GARcíA, G. (1975). Suelos de margas miocénicas de Murcia. Mineralogía, génesis y clasificación. Tesis Doctoral, Universidad de Granada, 186 pp. 
SÁnchez Soriano, A., Romero Díaz, A., Marín SANLEANDro, P. (2003). Procesos de piping en campos de cultivo abandonados (Campos del Río, Murcia). En: Control de la erosión y degradación del suelo (Bienes, R., Marques, M.J., Eds.). IMIA, 625-629 pp. Madrid.

SÁnchez Soriano, A., Marín SAnleAndro, P., Romero Díaz, A. (2004). The piping process in Murcia (S.E. Spain): II Effect of Physical Soil Properties. In: Fourth International Conference on Land Degradation (Faz, A., Ortiz, R., García, G., Eds.). Cartagena, Spain, Topic 4 (23). Cd Extended abstracts

Ternan, J.L., Elmes, A., FitzJohn, C. Y Willians, A.G. (1998). Piping susceptibility and the role of hydro-geomorphic controls in pipe development in alluvial sediments, Central Spain. Z. Geomorph. N.F., 42 (1): 75-87.

Vandekerckhove, L., Poesen, J., Oostwoud Wijdenes, D., Gyssels, G., BeuseLINCK, L., DE LUNA, E. (2000). Characteristics and controlling factors of bank gullies in two semi-arid Mediterranean environments. Geomorphology 33: 37-58.

WATTS, G. (1991). The relationship between soil piping and changing farming techniques on semiarid agricultural terraces. Proceeding of $20^{\text {th }}$ General Assembly of the International Union of Geodesy and Geophysics at Viene. IAHS Publication 202: 81-89. 